\title{
Comorbidity of Trauma
}

Ting-Shuo Huang

2017/02/28

\section{Load required packages}

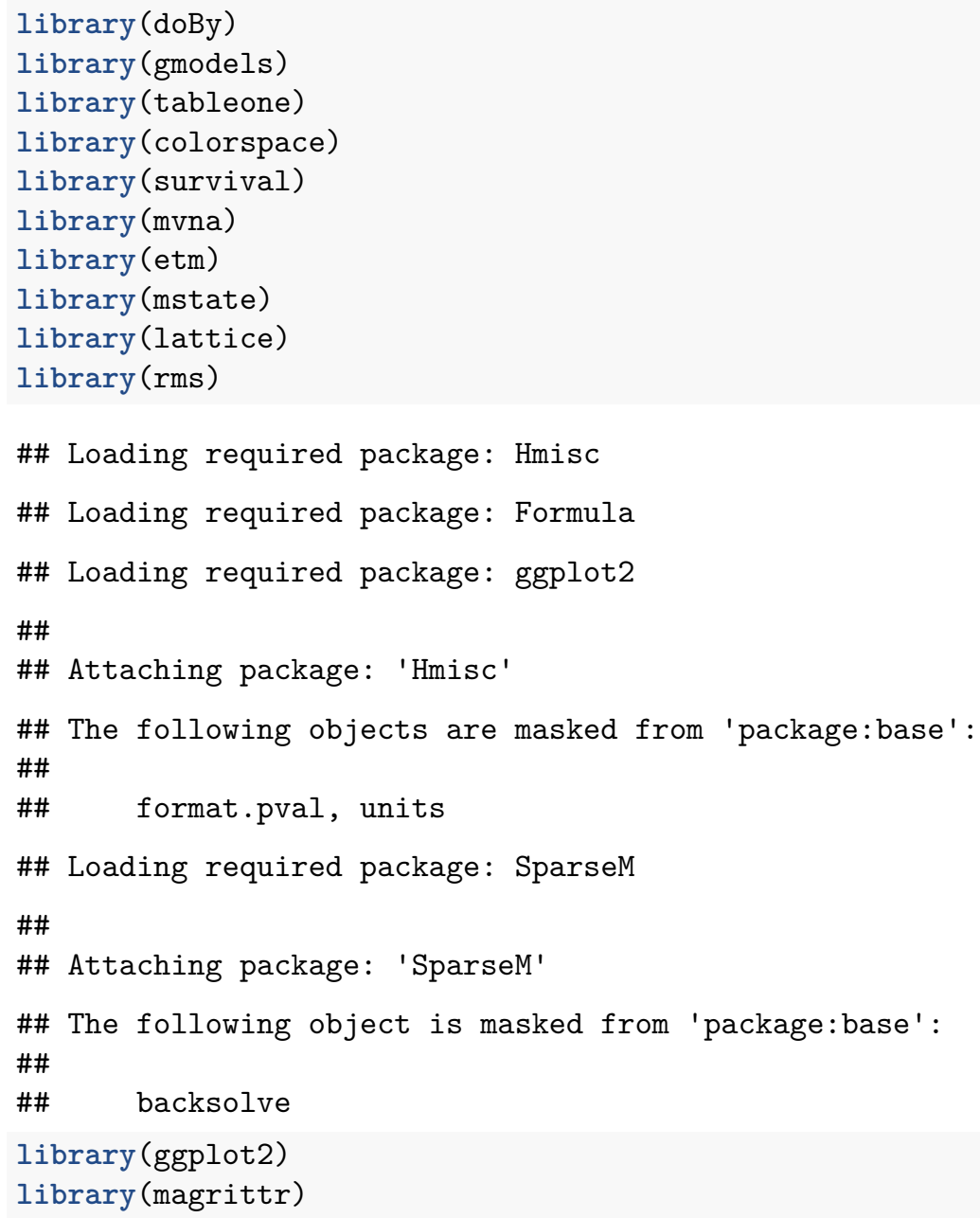

\section{Patient selection and prepare datasets (S1 Figure)}

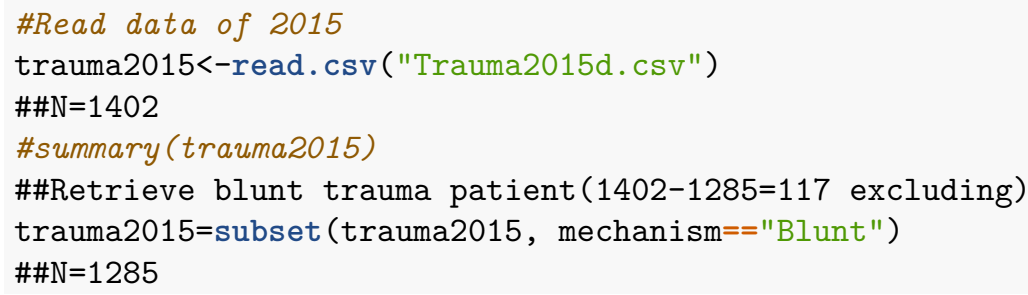




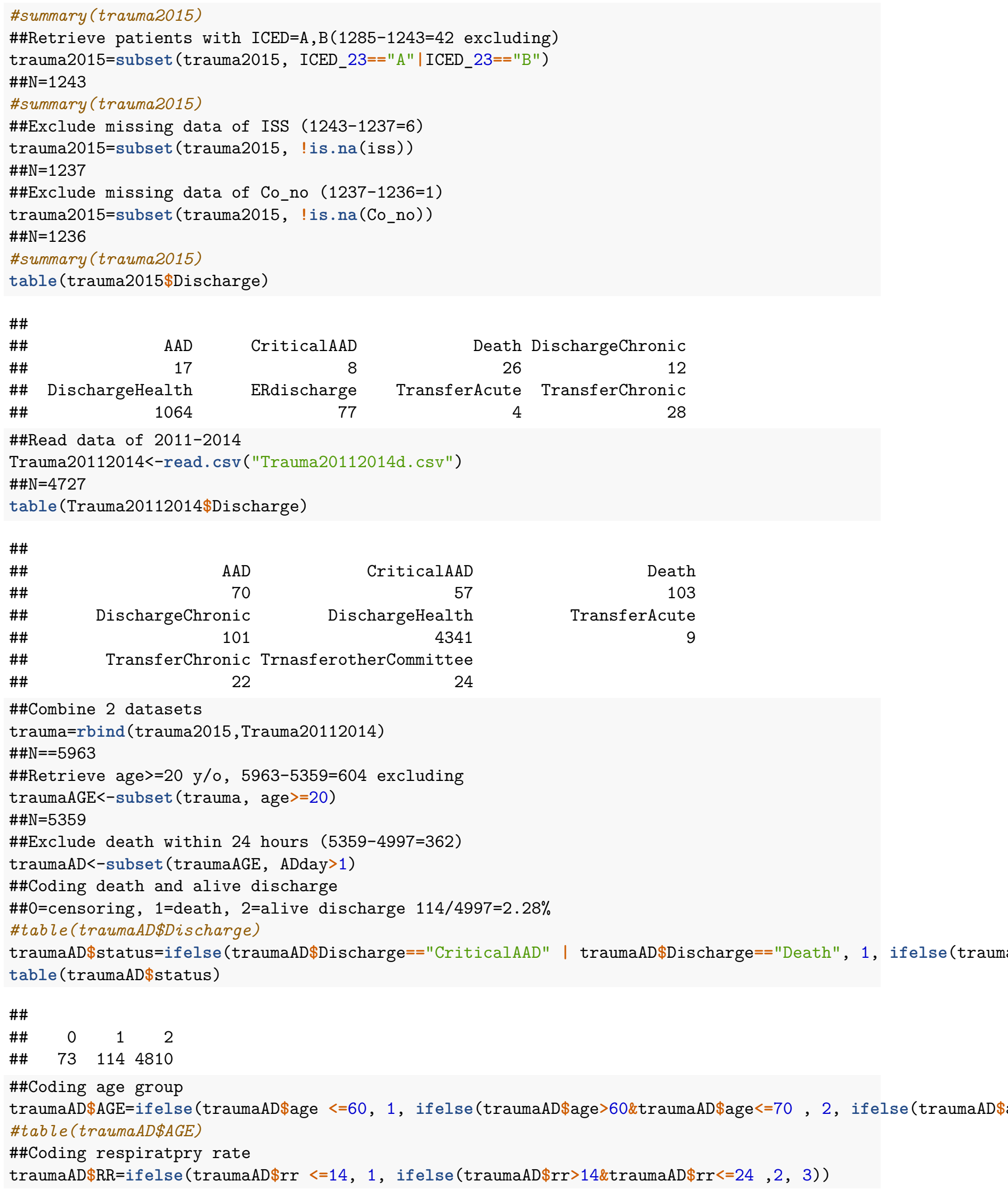




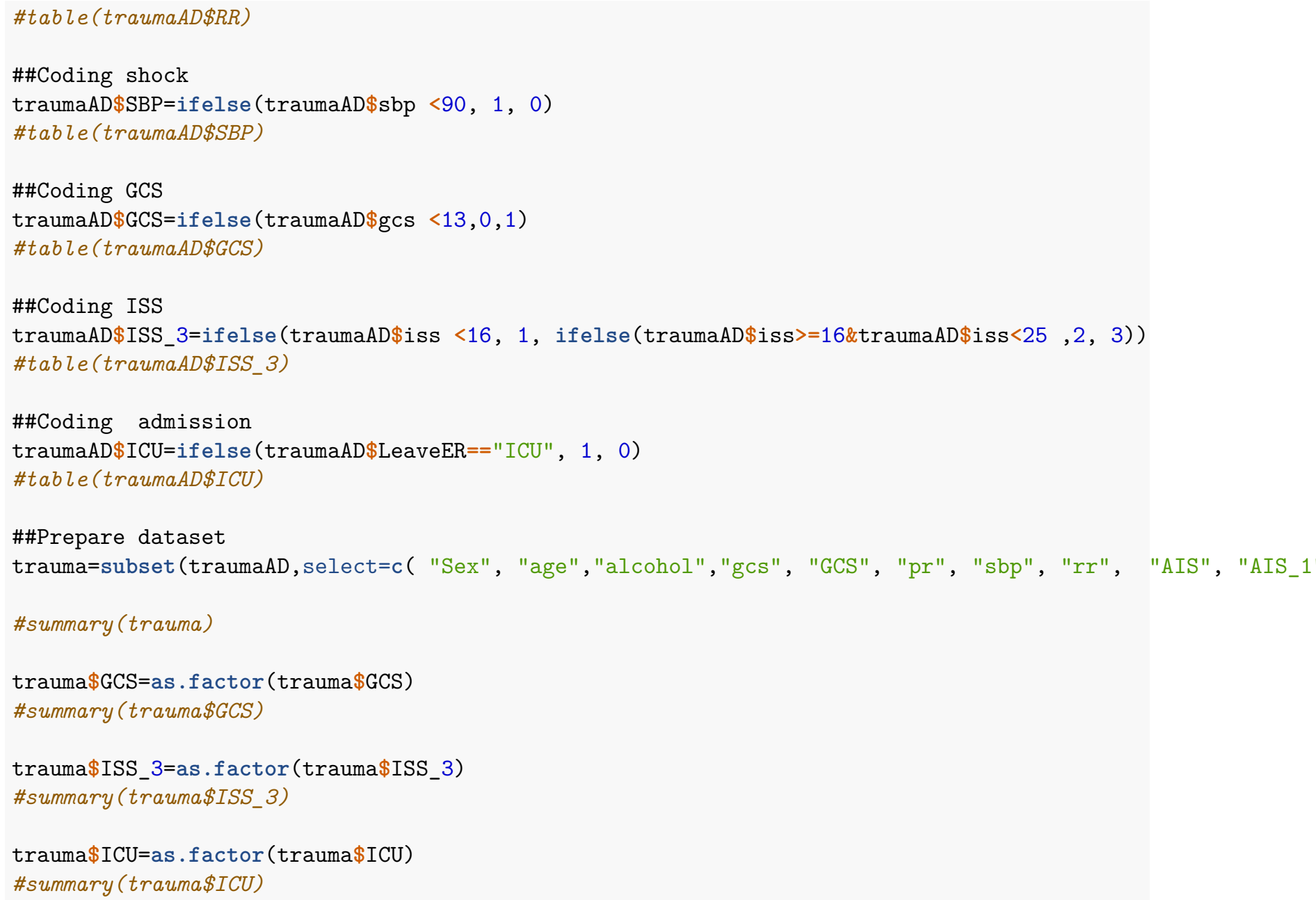

\section{Data exploration and create S1 Table and Table 1}

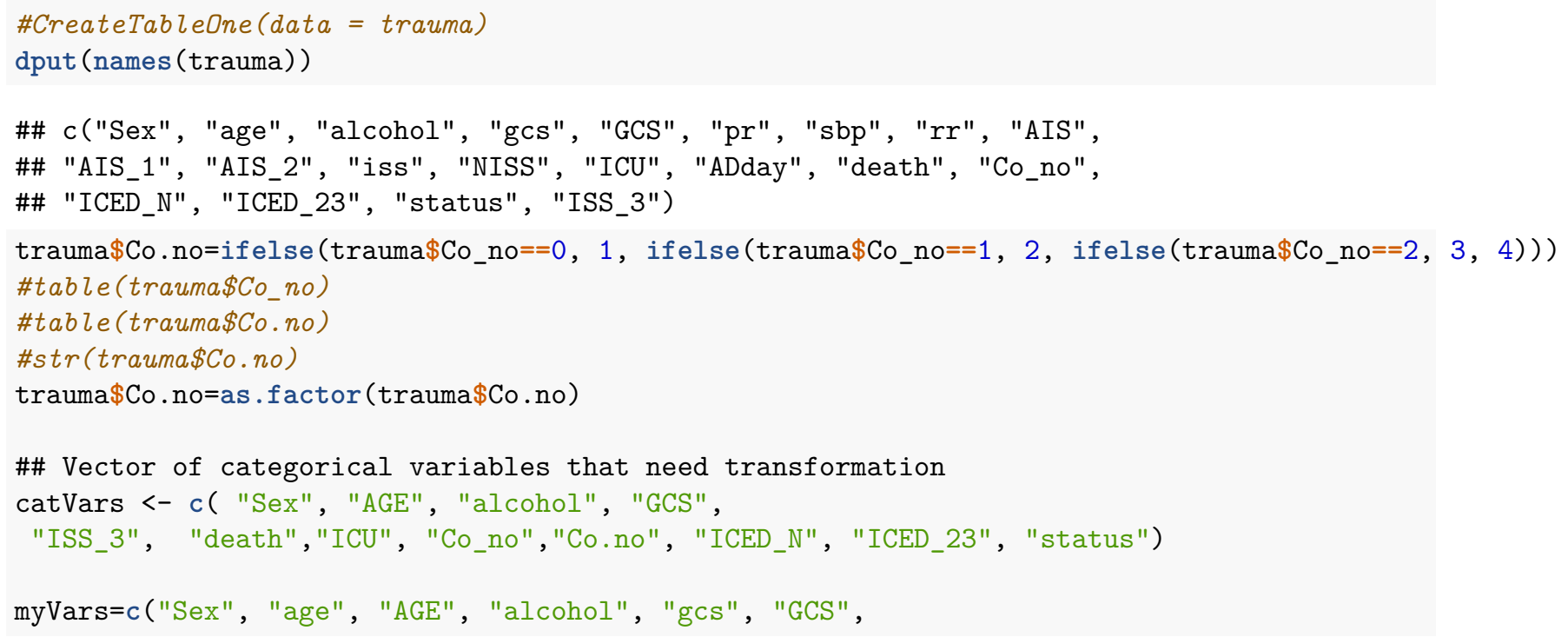




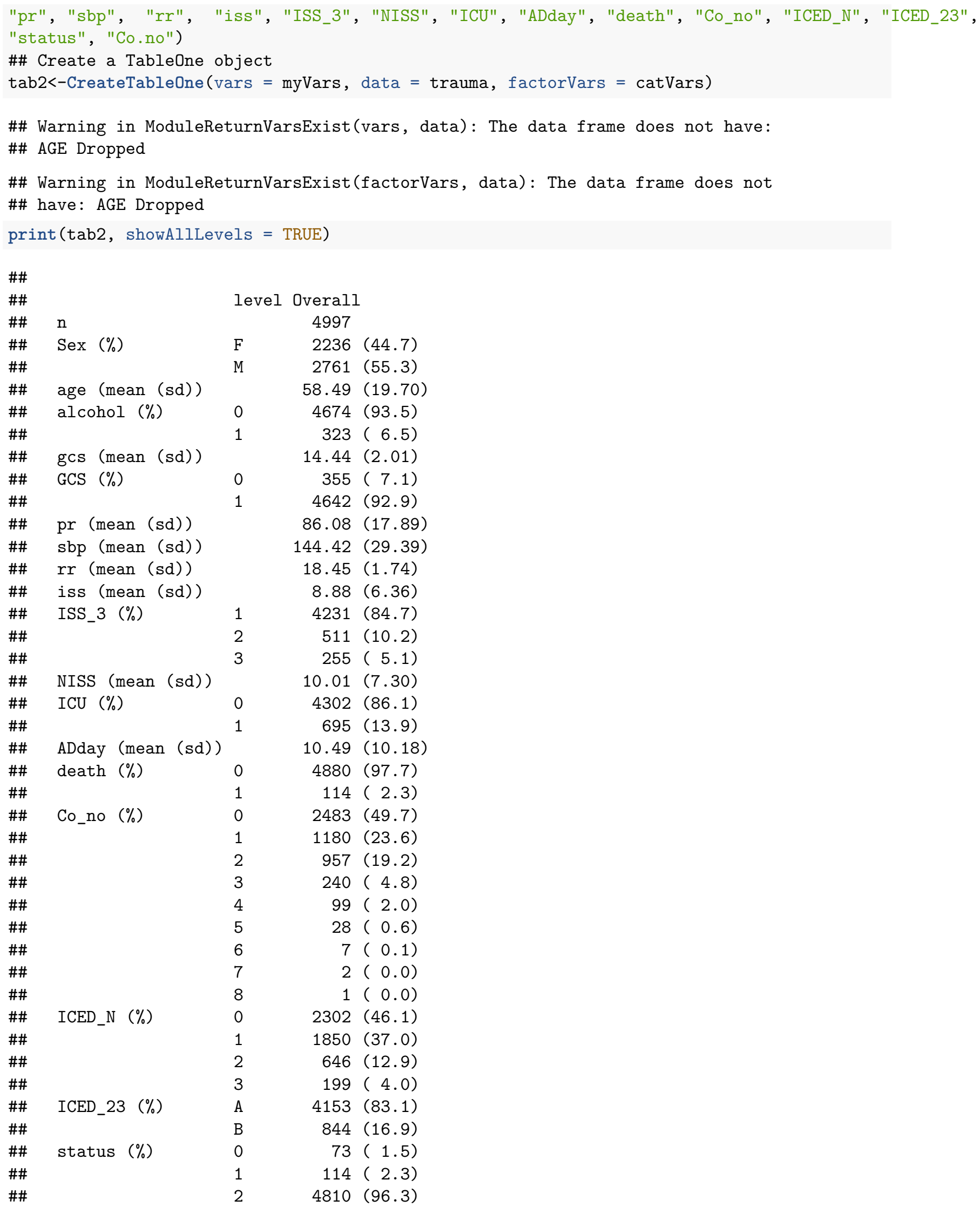




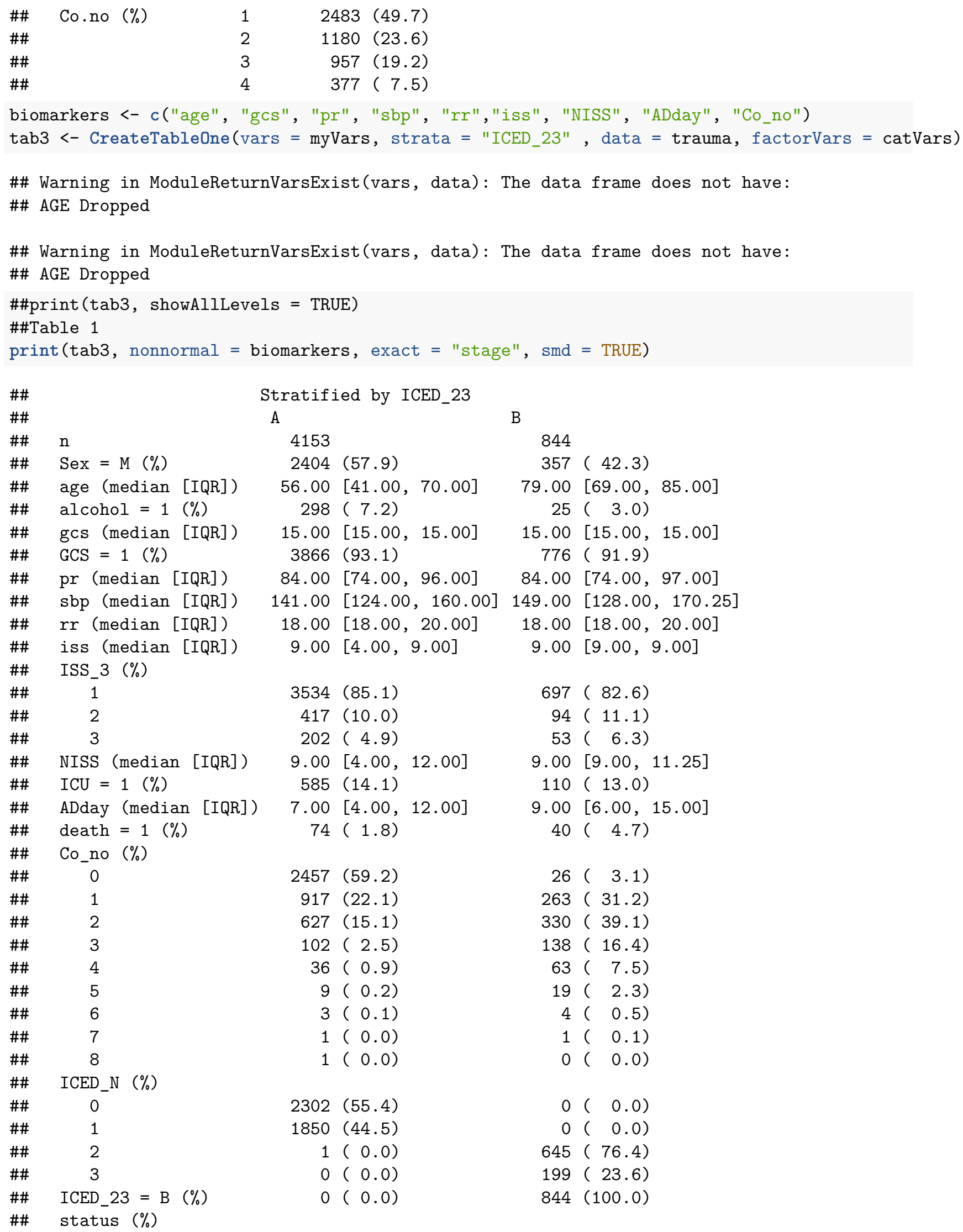

\#\#

\#\#

\#\# n

Stratified by ICED_23$$
\text { A }
$$ 


\begin{tabular}{|c|c|c|c|c|c|}
\hline \#\# & 0 & 57 & $(1.4)$ & & $16(1.9)$ \\
\hline \#\# & 1 & 74 & $(1.8)$ & & $40(4.7)$ \\
\hline \#\# & 2 & 4022 & $(96.8)$ & & $788(93.4)$ \\
\hline \#\# & Co.no $(\%)$ & & & & \\
\hline \#\# & 1 & 2457 & $(59.2)$ & & $26(3.1)$ \\
\hline \#\# & 2 & 917 & $(22.1)$ & & $263(31.2)$ \\
\hline \#\# & 3 & 627 & $(15.1)$ & & $330(39.1)$ \\
\hline \#\# & 4 & 152 & $(3.7)$ & & $225(26.7)$ \\
\hline \#\# & & Stratifi & ied by $\mathrm{IC}$ & $E D \_23$ & \\
\hline \#\# & & $\mathrm{p}$ & test & SMD & \\
\hline \#\# & $\mathrm{n}$ & & & & \\
\hline \#\# & $\operatorname{Sex}=M(\%)$ & $<0.001$ & & 0.316 & \\
\hline \#\# & age (median [IQR]) & $<0.001$ & nonnorm & 1.313 & \\
\hline \#\# & alcohol = $1(\%)$ & $<0.001$ & & 0.193 & \\
\hline \#\# & gcs (median [IQR]) & 0.003 & nonnorm & 0.012 & \\
\hline \#\# & $\mathrm{GCS}=1(\%)$ & 0.268 & & 0.044 & \\
\hline \#\# & $\mathrm{pr}$ (median [IQR]) & 0.381 & nonnorm & 0.050 & \\
\hline \#\# & $\operatorname{sbp}(\operatorname{median}[\mathrm{IQR}])$ & $<0.001$ & nonnorm & 0.205 & \\
\hline \#\# & $\operatorname{rr}(\operatorname{median}[\mathrm{IQR}])$ & 0.911 & nonnorm & 0.024 & \\
\hline \#\# & iss (median [IQR]) & $<0.001$ & nonnorm & 0.207 & \\
\hline \#\# & ISS_3 (\%) & 0.129 & & 0.074 & \\
\hline \#\# & 1 & & & & \\
\hline \#\# & 2 & & & & \\
\hline \#\# & 3 & & & & \\
\hline \#\# & NISS (median [IQR]) & $<0.001$ & nonnorm & 0.118 & \\
\hline \#\# & $\mathrm{ICU}=1(\%)$ & 0.452 & & 0.031 & \\
\hline \#\# & ADday (median [IQR]) & $<0.001$ & nonnorm & 0.259 & \\
\hline \#\# & death $=1(\%)$ & $<0.001$ & & 0.167 & \\
\hline \#\# & Co_no $(\%)$ & $<0.001$ & & 1.637 & \\
\hline \#\# & 0 & & & & \\
\hline \#\# & 1 & & & & \\
\hline \#\# & 2 & & & & \\
\hline \#\# & 3 & & & & \\
\hline \#\# & 4 & & & & \\
\hline \#\# & 5 & & & & \\
\hline \#\# & 6 & & & & \\
\hline \#\# & 7 & & & & \\
\hline \#\# & 8 & & & & \\
\hline \#\# & ICED_N $(\%)$ & $<0.001$ & & 91.130 & \\
\hline \#\# & 0 & & & & \\
\hline \#\# & 1 & & & & \\
\hline \#\# & 2 & & & & \\
\hline \#\# & 3 & & & & \\
\hline \#\# & ICED_23 = B (\%) & $<0.001$ & & $\mathrm{NaN}$ & \\
\hline \#\# & status $(\%)$ & $<0.001$ & & 0.173 & \\
\hline \#\# & 0 & & & & \\
\hline \#\# & 1 & & & & \\
\hline \#\# & 2 & & & & \\
\hline \#\# & Co.no $(\%)$ & $<0.001$ & & 1.636 & \\
\hline \#\# & 1 & & & & \\
\hline \#\# & 2 & & & & \\
\hline \#\# & 3 & & & & \\
\hline \#\# & 4 & & & & \\
\hline
\end{tabular}



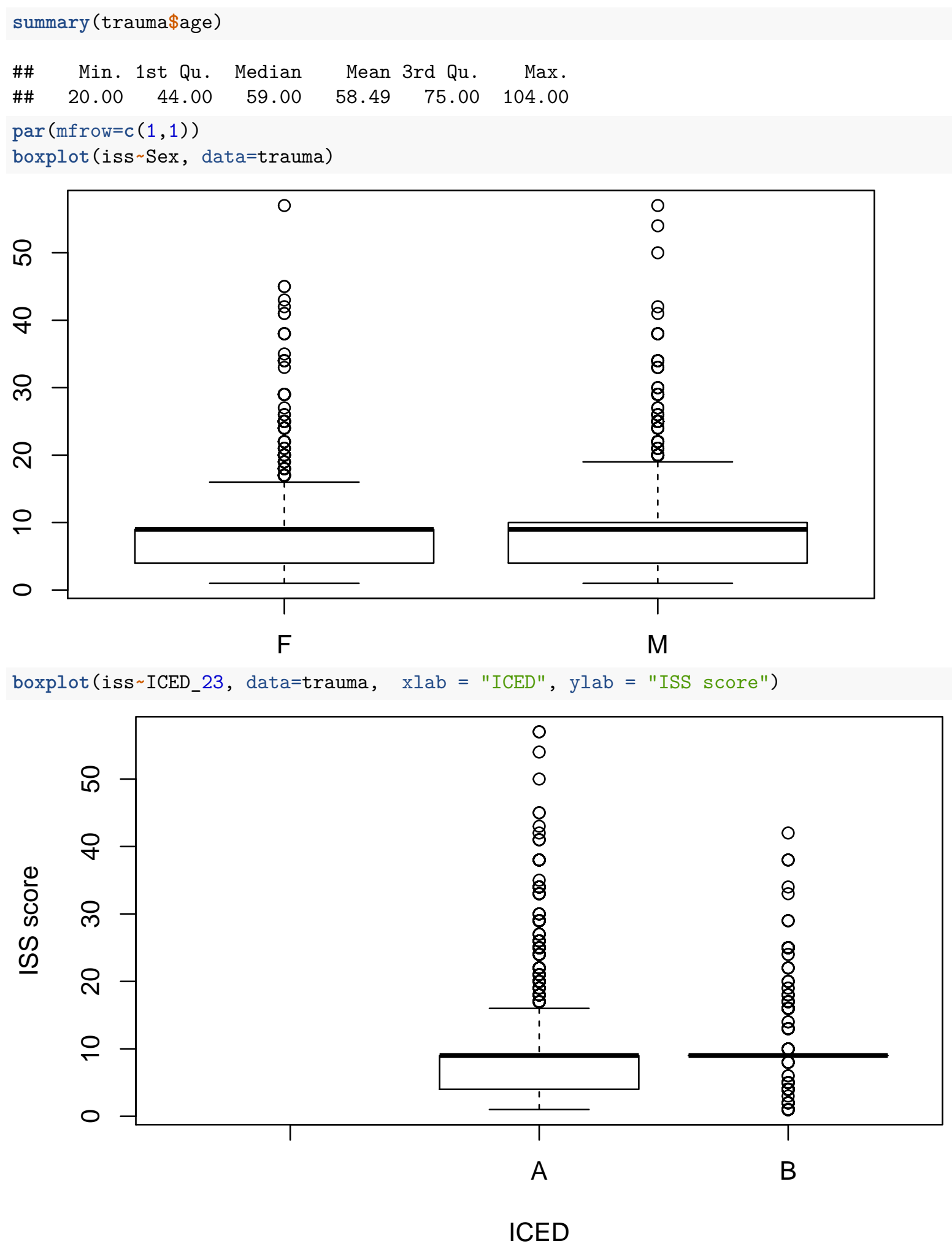

CrossTable (trauma $\$$ ISS_3, trauma $\$$ Sex, chisq $=\mathrm{T}$, fisher $=\mathrm{F}$ ) 


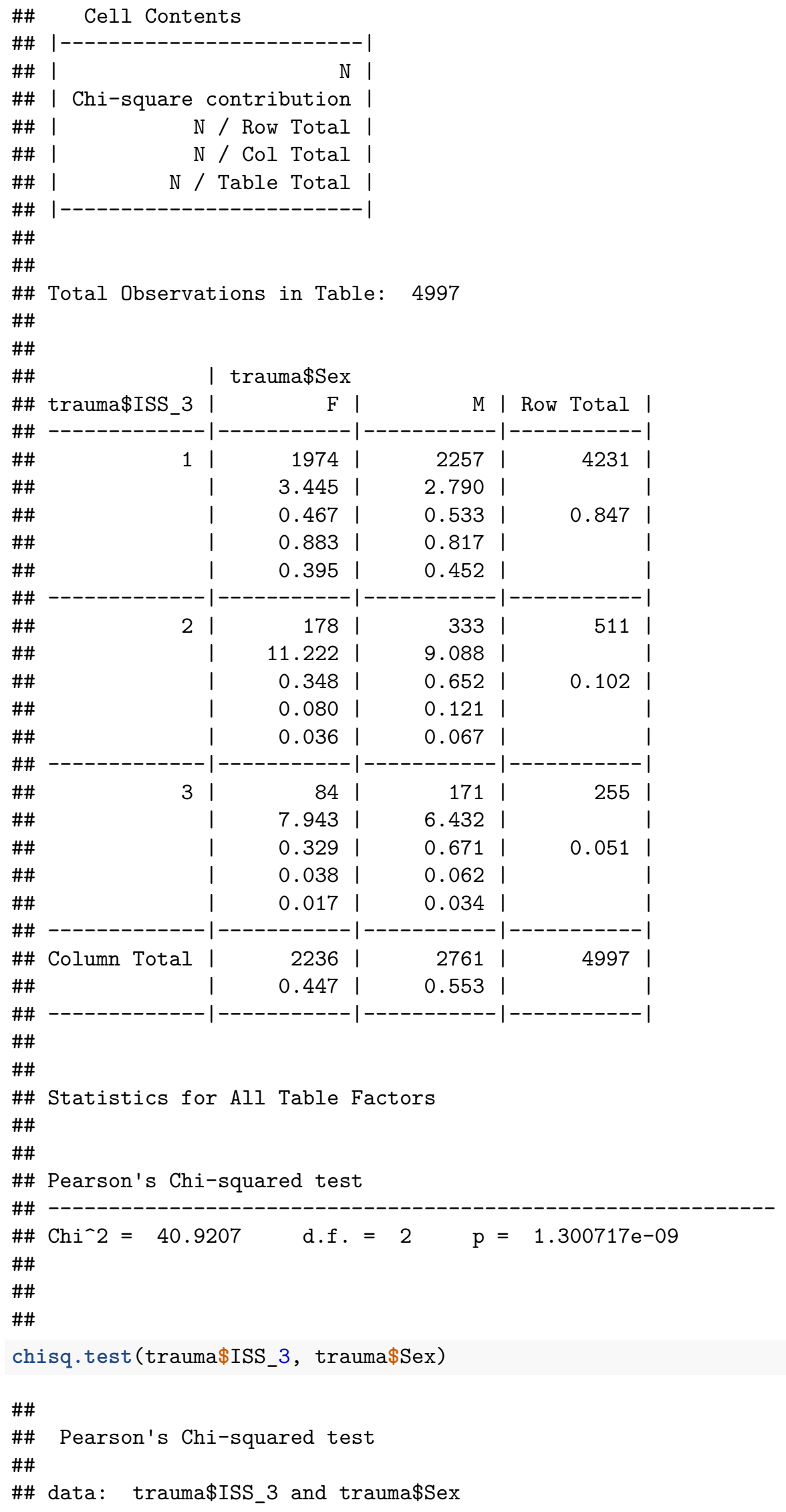




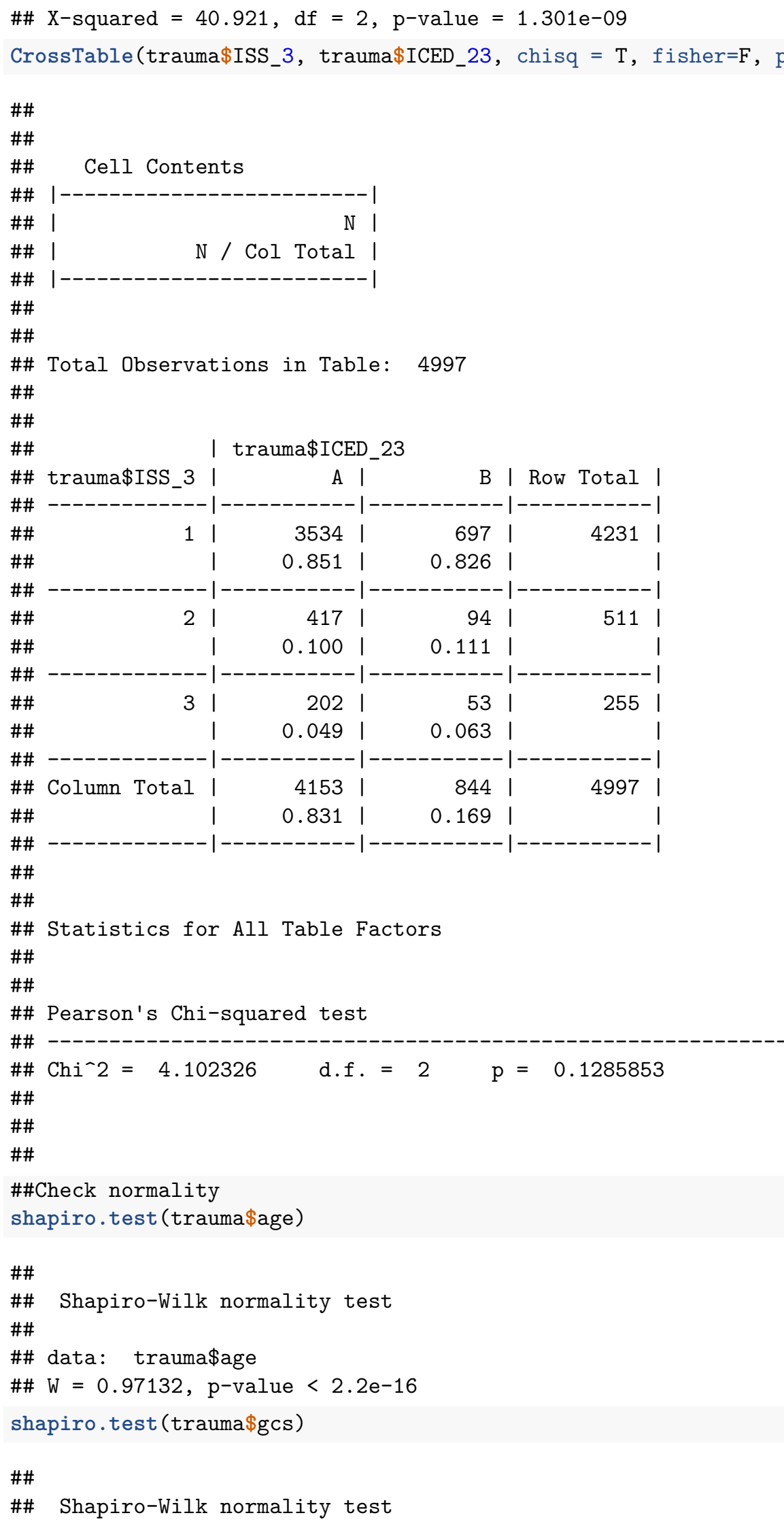




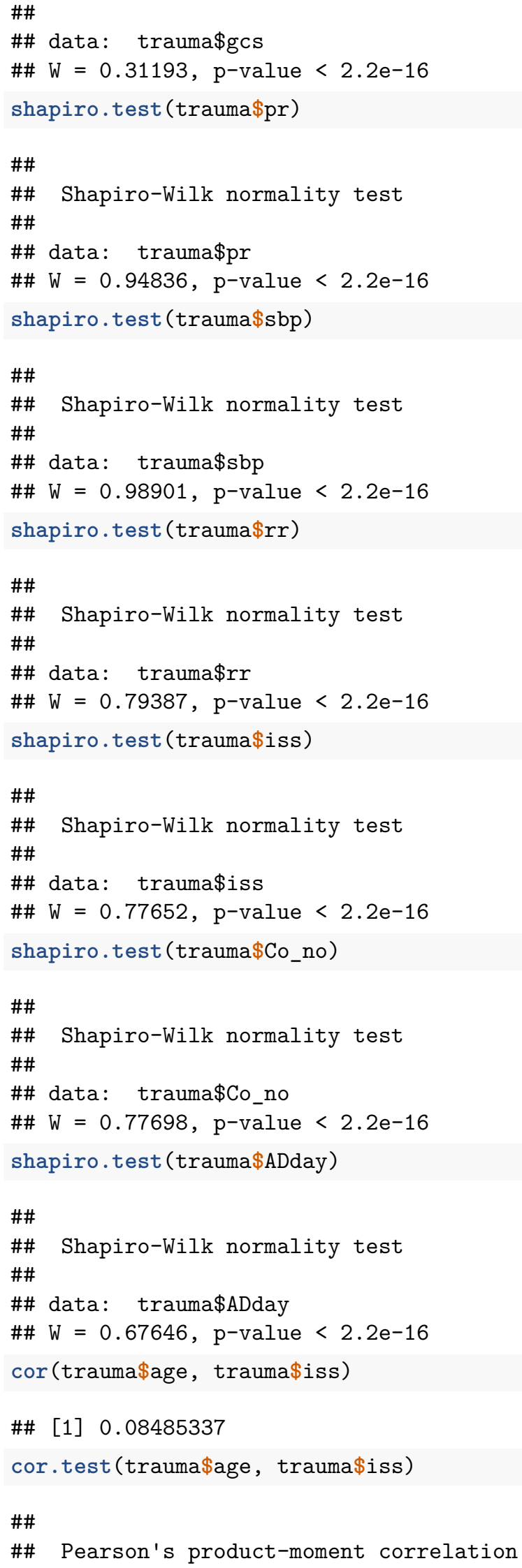


\#\# data: trauma\$age and trauma\$iss

$\# \# \mathrm{t}=6.0187, \mathrm{df}=4995, \mathrm{p}$-value $=1.883 \mathrm{e}-09$

\#\# alternative hypothesis: true correlation is not equal to 0

\#\# 95 percent confidence interval:

\#\# $0.05726048 \quad 0.11231673$

\#\# sample estimates:

\#\# cor

\#\# 0.08485337

\section{Prepare counting process dataset for competing risk model}

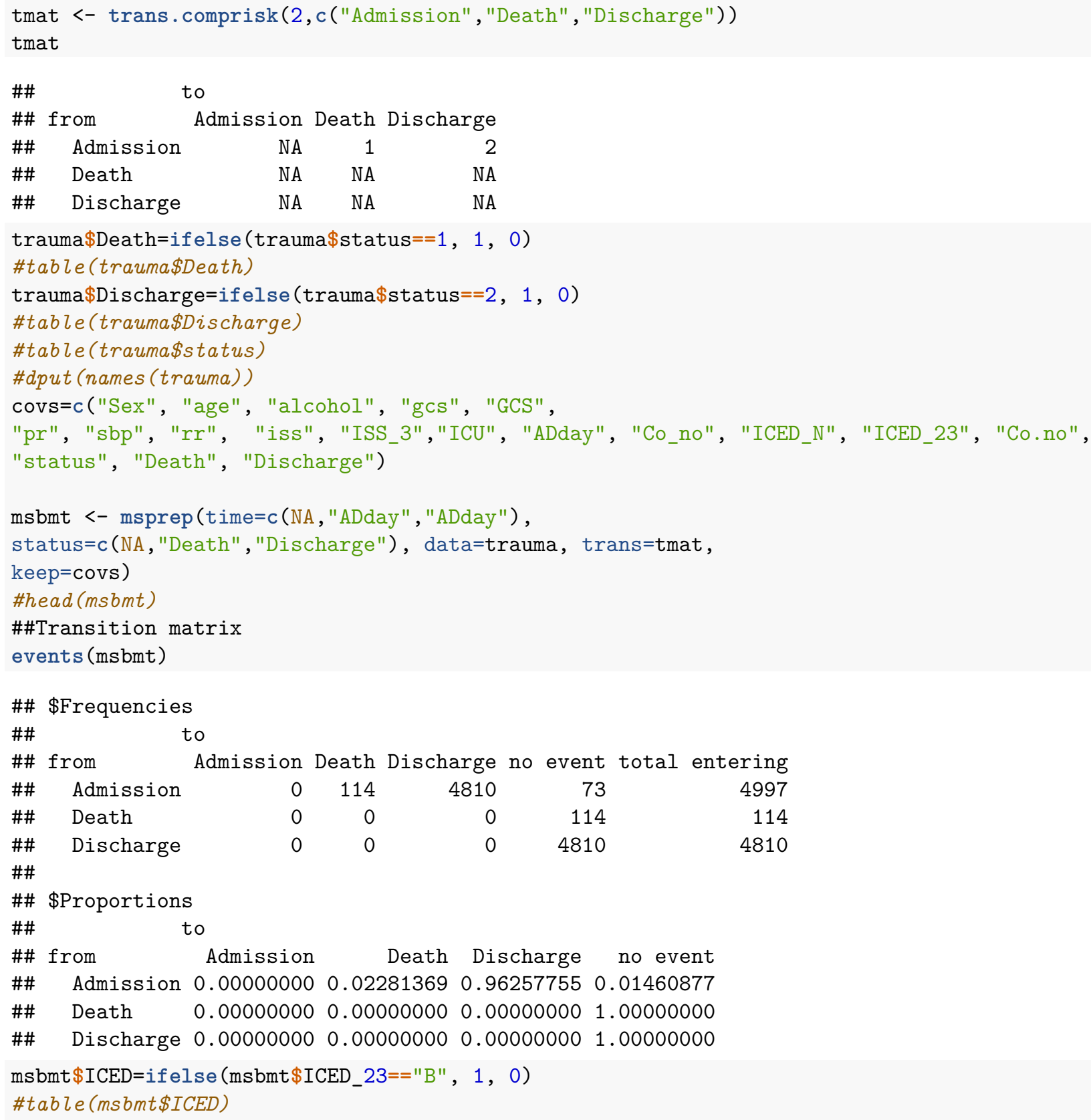


\#\#Expand covariate

COV <-c( "Sex", "age", "alcohol", "GCS", "gcs", "sbp", "ISS_3","iss", "ICU", "Co_no","Co.no", "ICED")

sdata<- expand.covs (msbmt, COV, append=TRUE, Iongnames=FALSE)

\#head (sdata)

\#dput (names (sdata))

\section{Nonparametric estimate of cumulative hazard function}

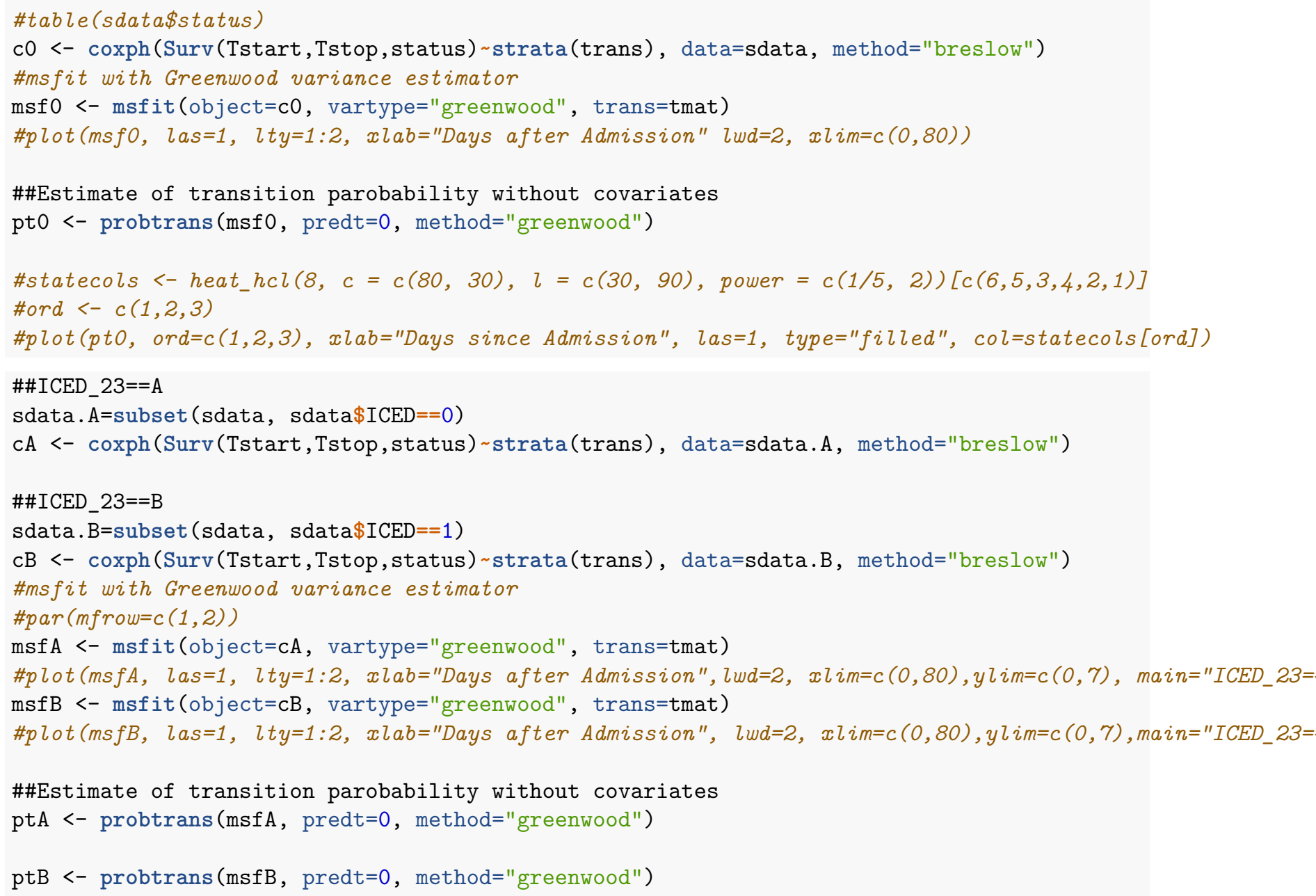

\section{Create Figure 1}

$\operatorname{msf} . A=m s f A[[1]]$

$m s f . B=m s f B[[1]]$

$\operatorname{par}(\operatorname{mfrow}=\mathrm{c}(1,2))$

plot (msf.A\$time [msf.A\$trans==1], msf.A\$Haz [msf.A\$trans==1], type="s", ylim=c $(0,1.0), x l i m=c(0,80), y l a b=$ lines (msf. $\mathrm{B} \$$ time [msf. $\mathrm{B} \$$ trans $==1]$, msf . $\mathrm{B} \$ \mathrm{Haz}[\mathrm{msf} . \mathrm{B} \$$ trans $==1]$, 
type="s", ylim=c(0,1.0), xlim=c $(0,80), \operatorname{col}="$ blue", main="Transition to death", cex=1, $1 w d=2)$

legend("topleft", col=c("blue", "orange"), $l$ ty=c $(1,1), 1 w d=c(2,2)$,

legend=c("ICED 2/3", "ICED 0/1"), cex=1 )

plot (msf .A\$time [msf .A\$trans==2], msf .A\$Haz [msf . A $\$$ trans $==2]$,

type="s", ylim=c(0,7.0), xlim=c(0,80), ylab="Cumulative hazards", xlab="Days since admission", col lines (msf . $B \$$ time $[\mathrm{msf} . \mathrm{B} \$$ trans $==2]$, msf . $B \$$ Haz [msf . $B \$$ trans $==2]$,

type="s", ylim=c $(0,1.0)$, xlim=c $(0,80)$, col="blue", main="Transplant to Death",cex=1, lwd=2)

legend("topleft", col=c("blue", "orange"), lty=c(1,1), lwd = c $(2,2)$,

legend=c("ICED 2/3", "ICED 0/1"), cex=1)

Transition to death

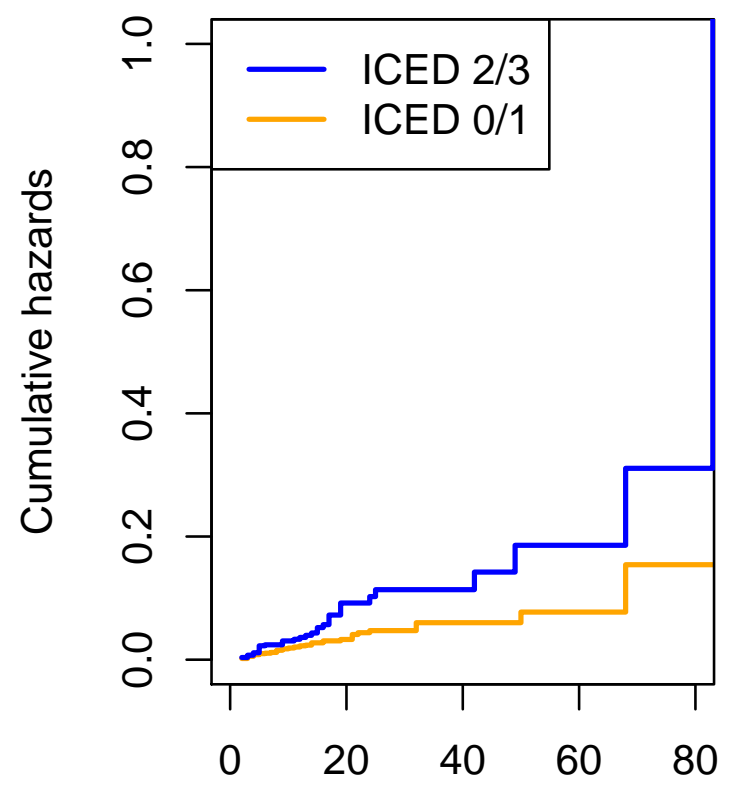

Days since admission

\section{Transition to discharge}

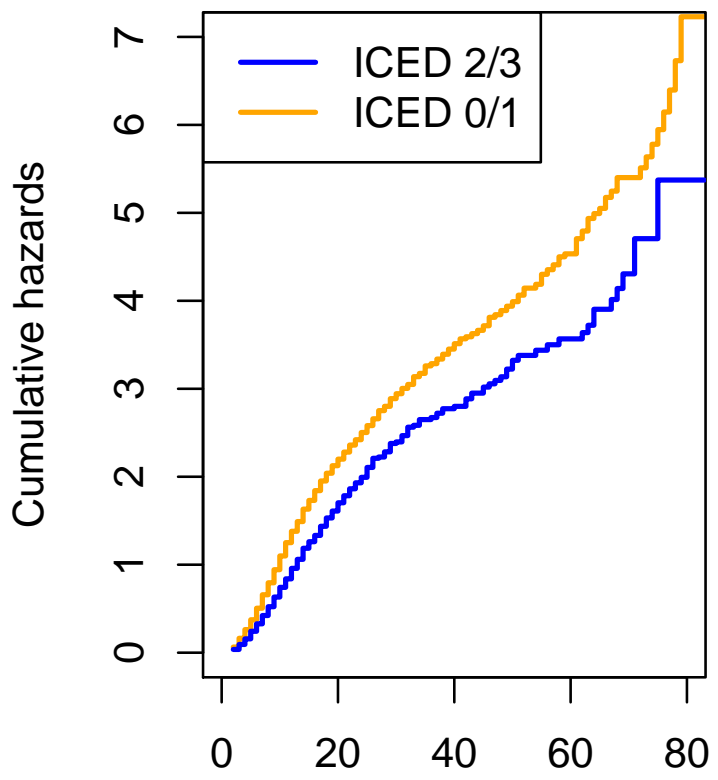

Days since admission

\section{Create Table 2}

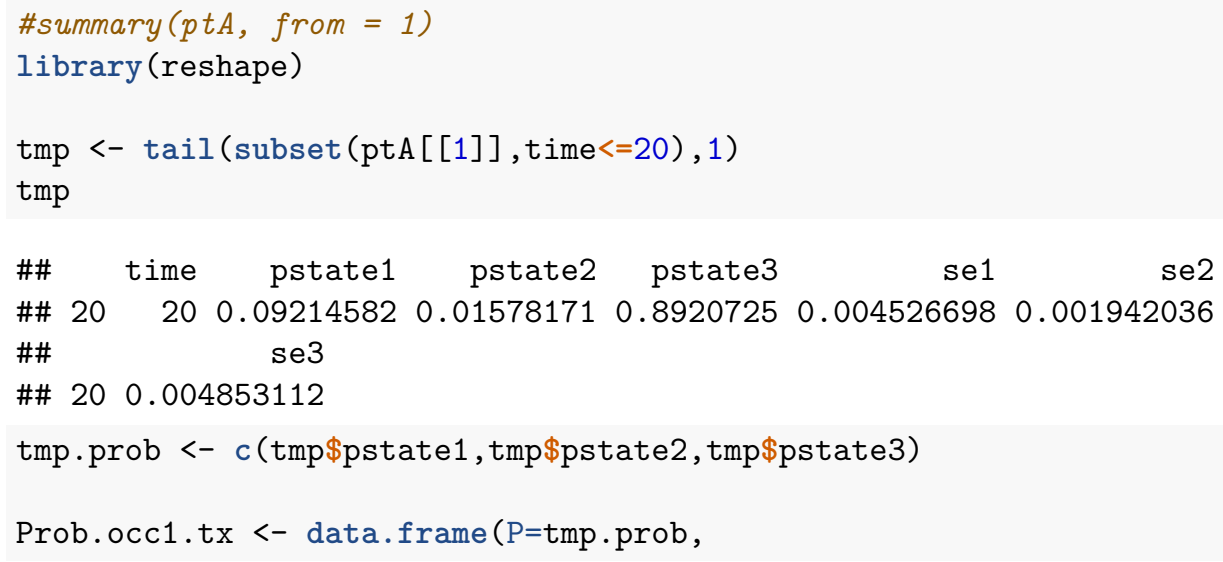




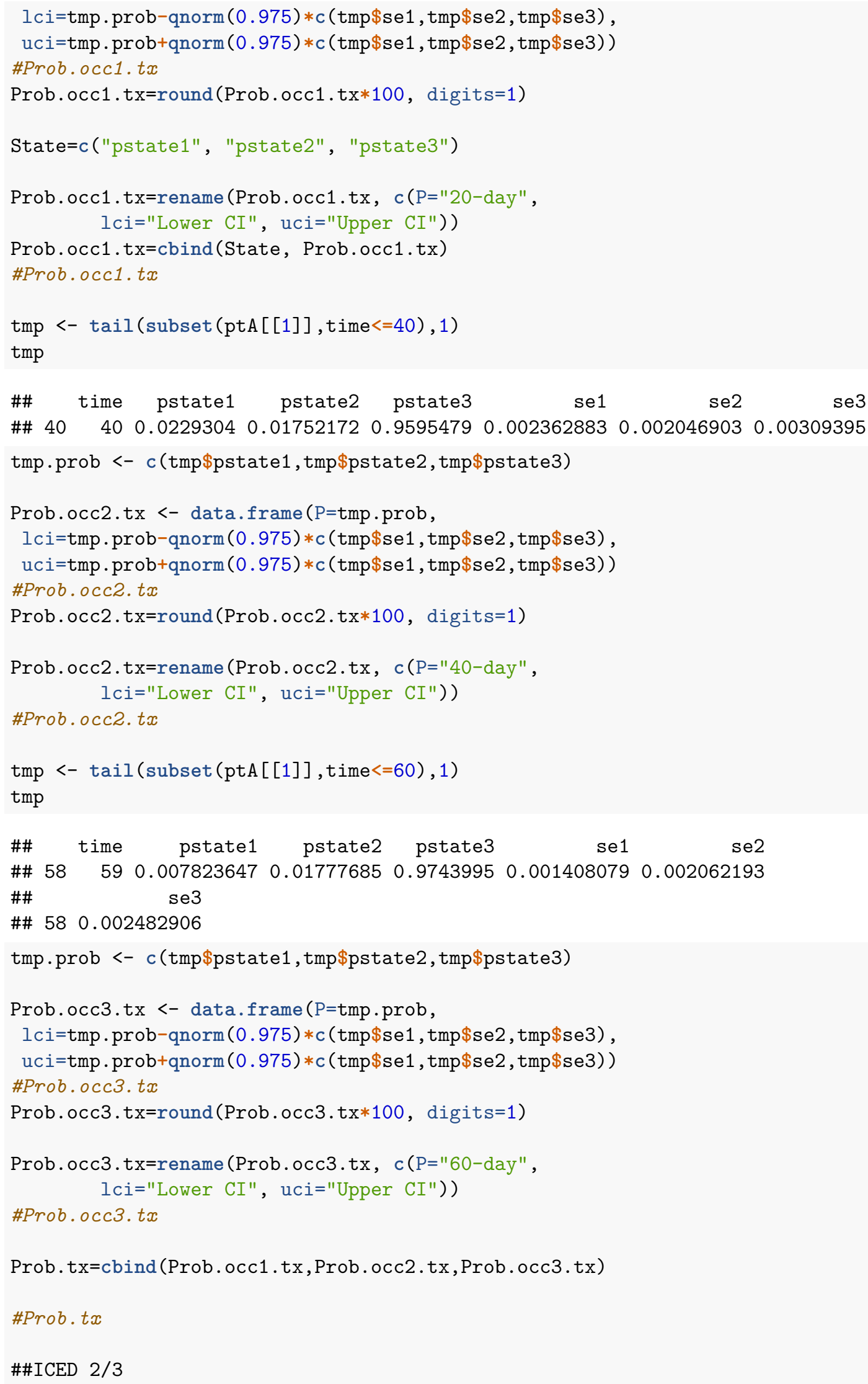




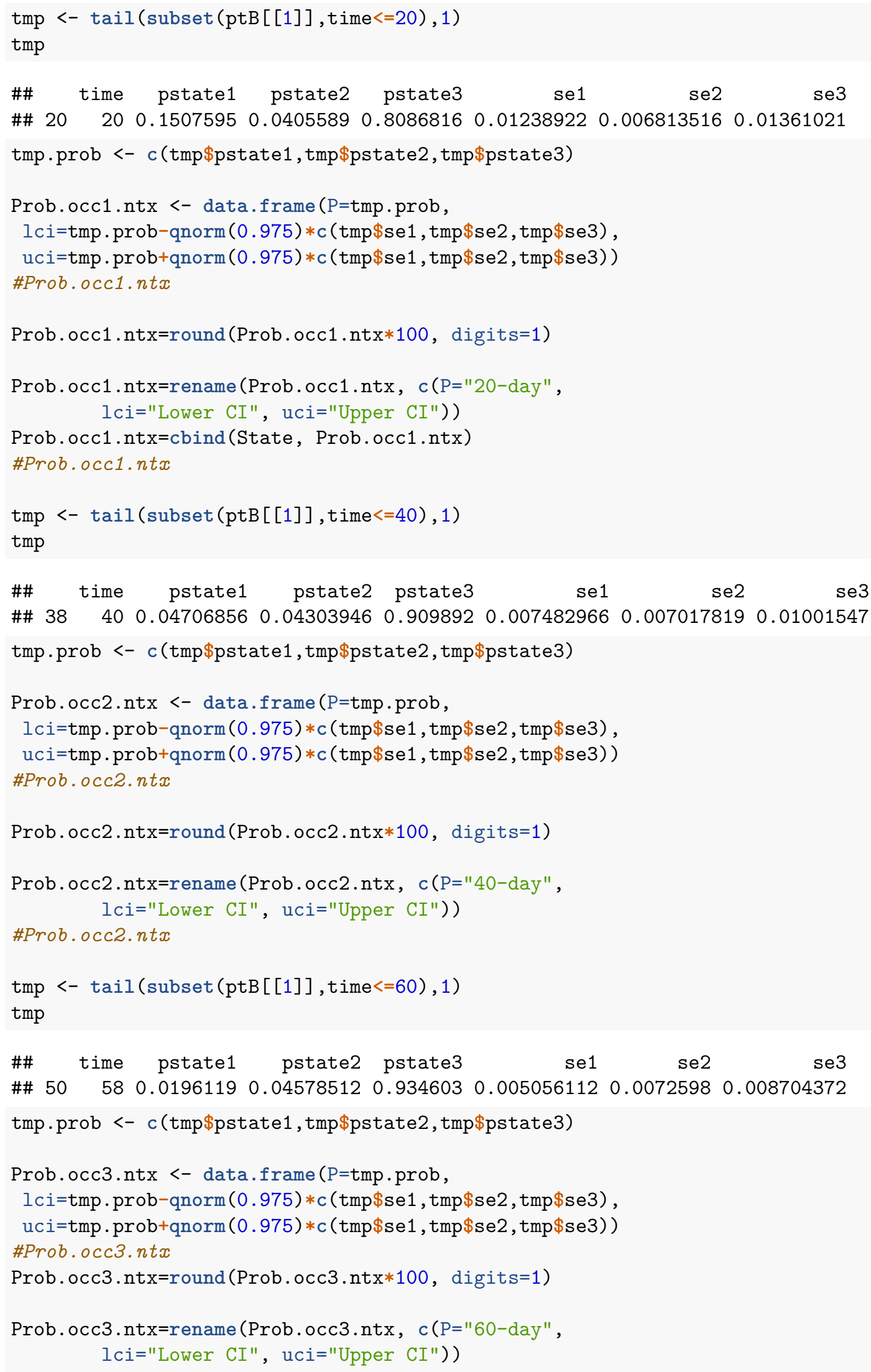




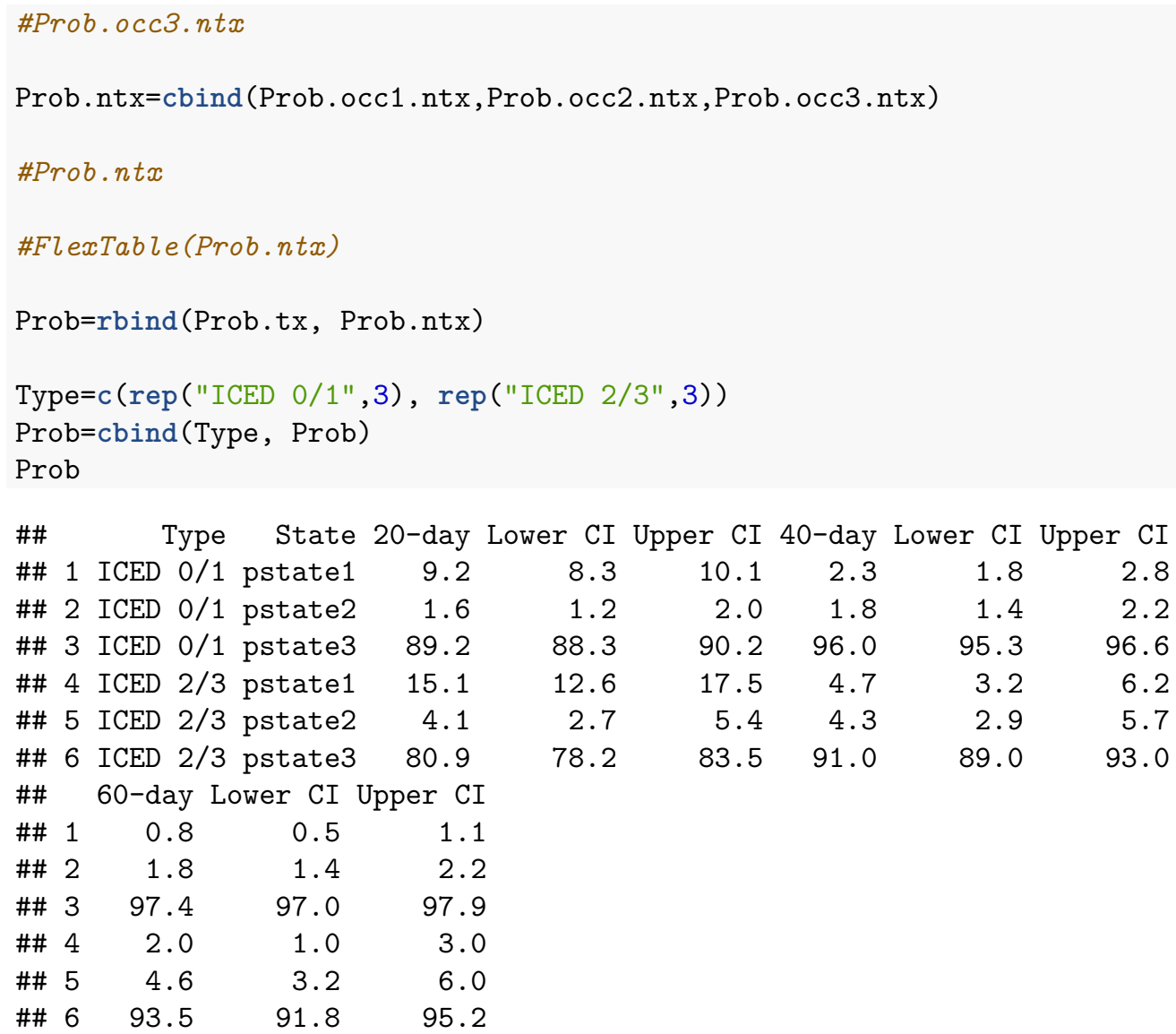

\section{Cause-specific Cox model}

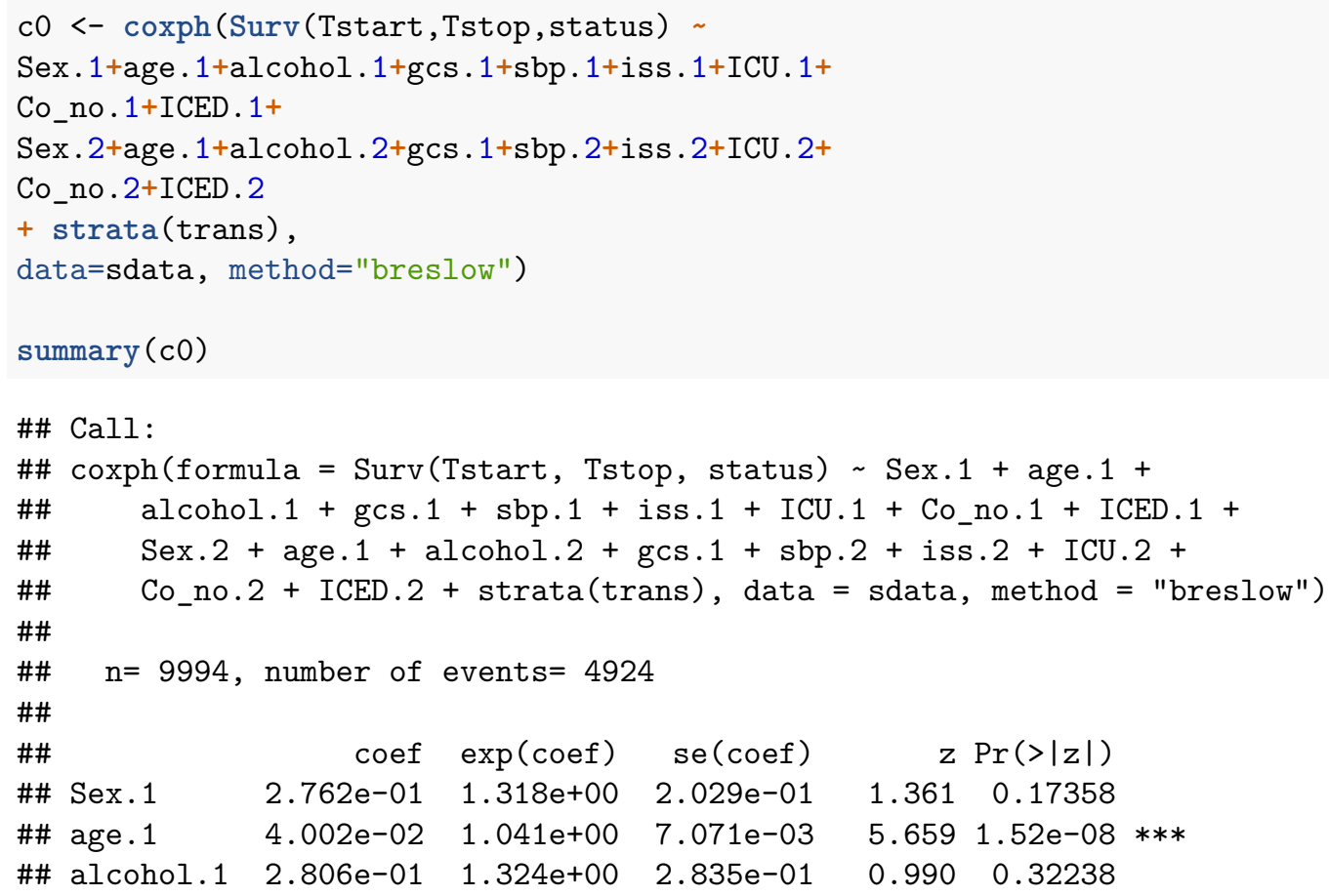




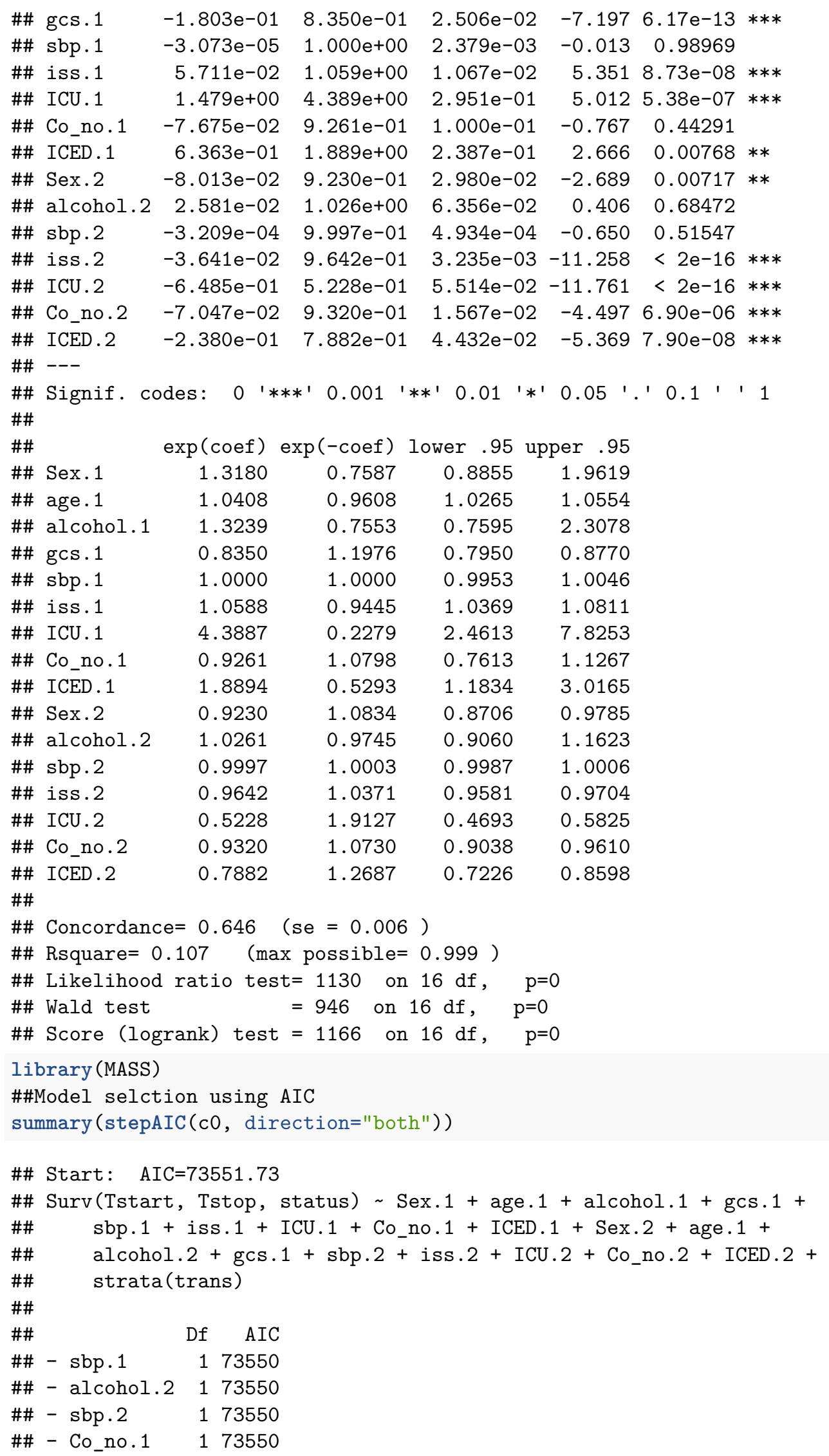




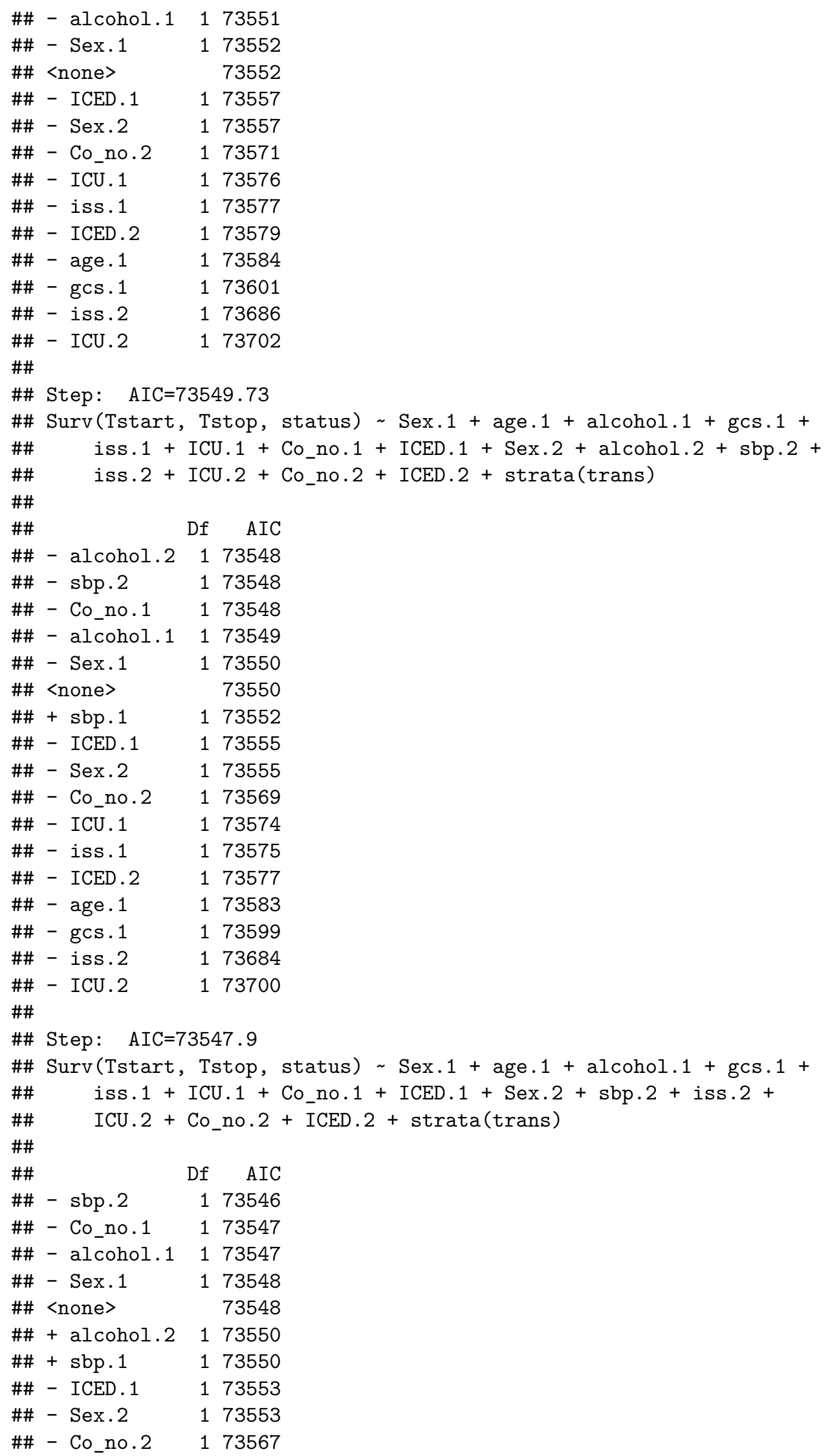




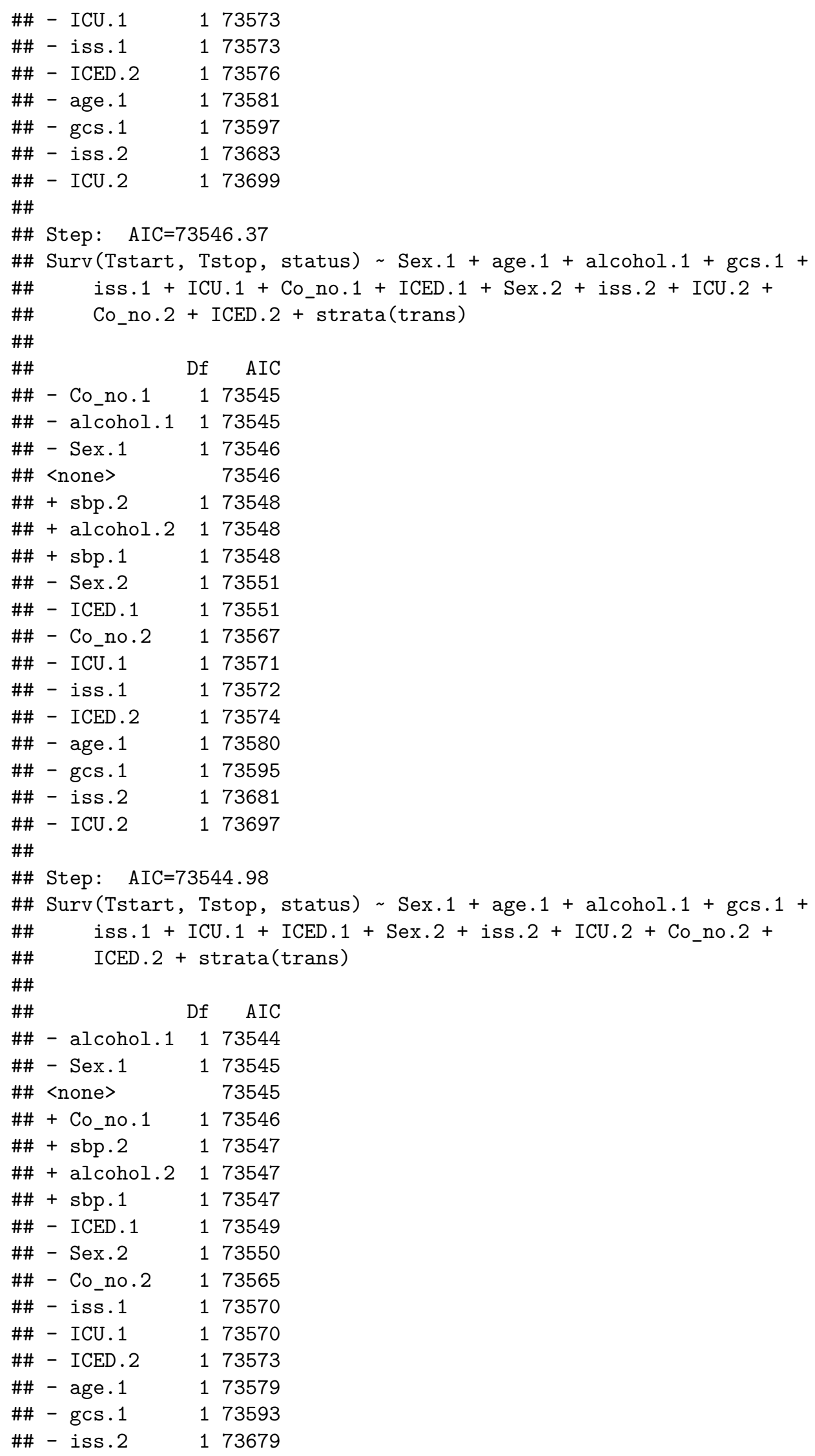




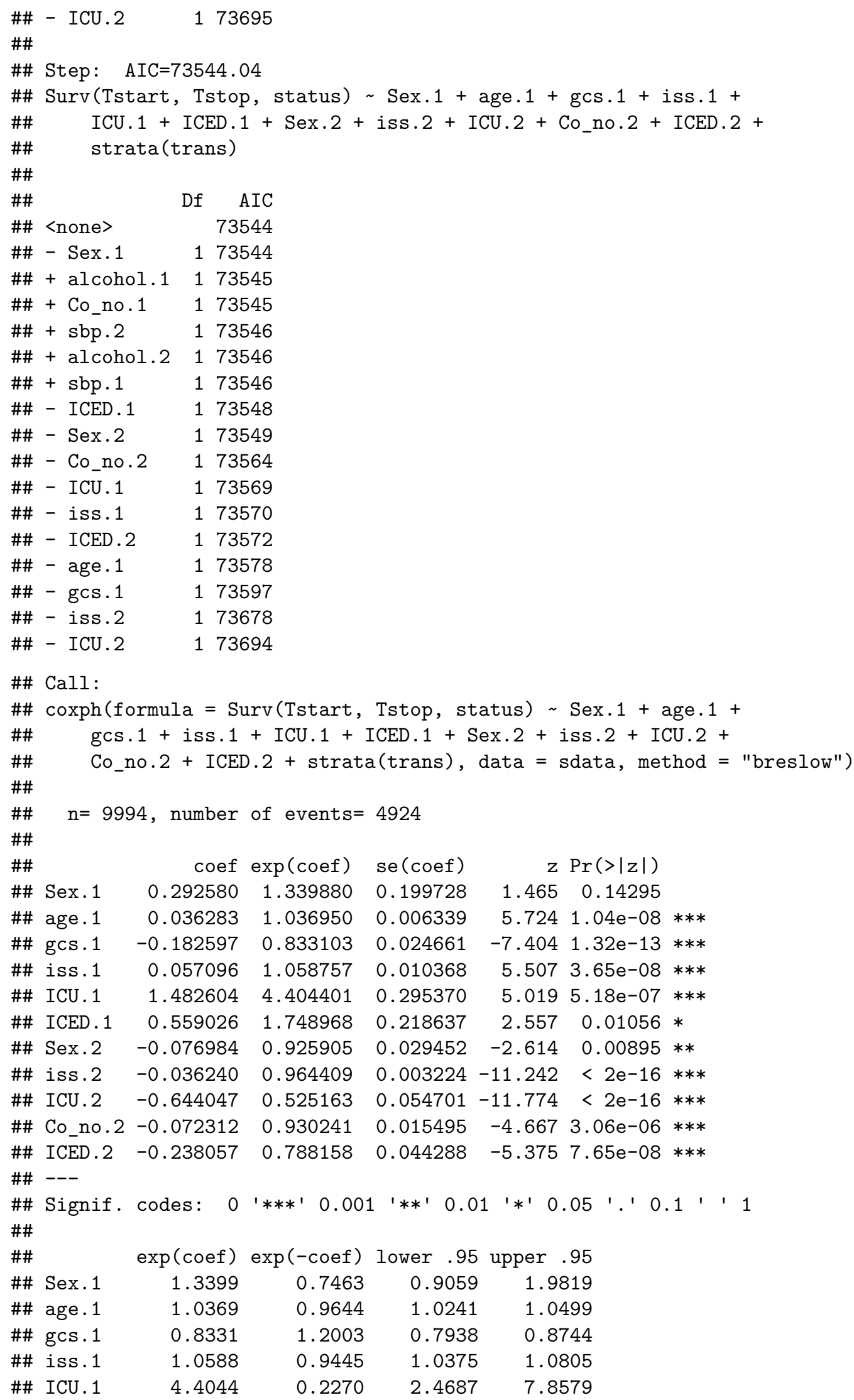




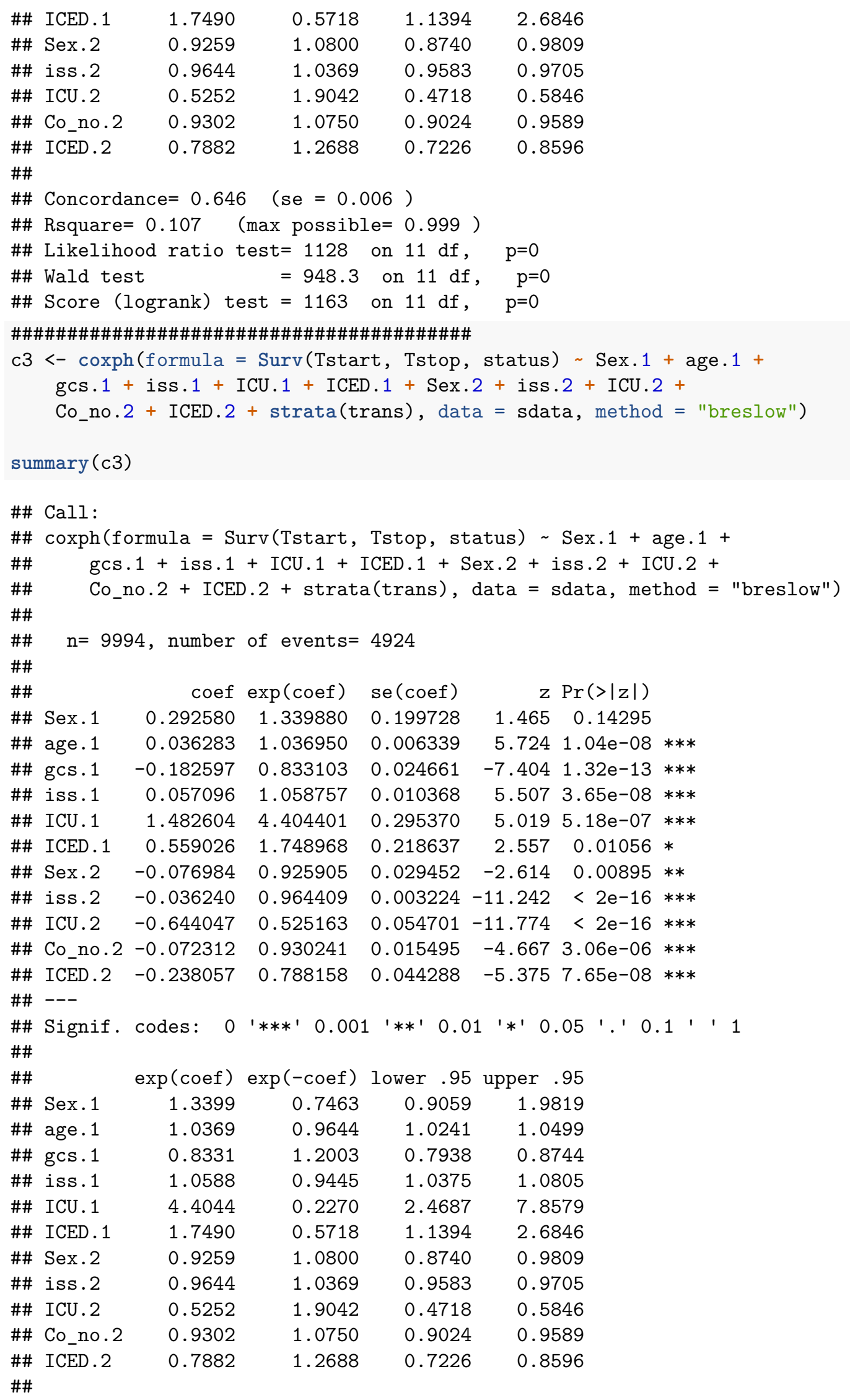




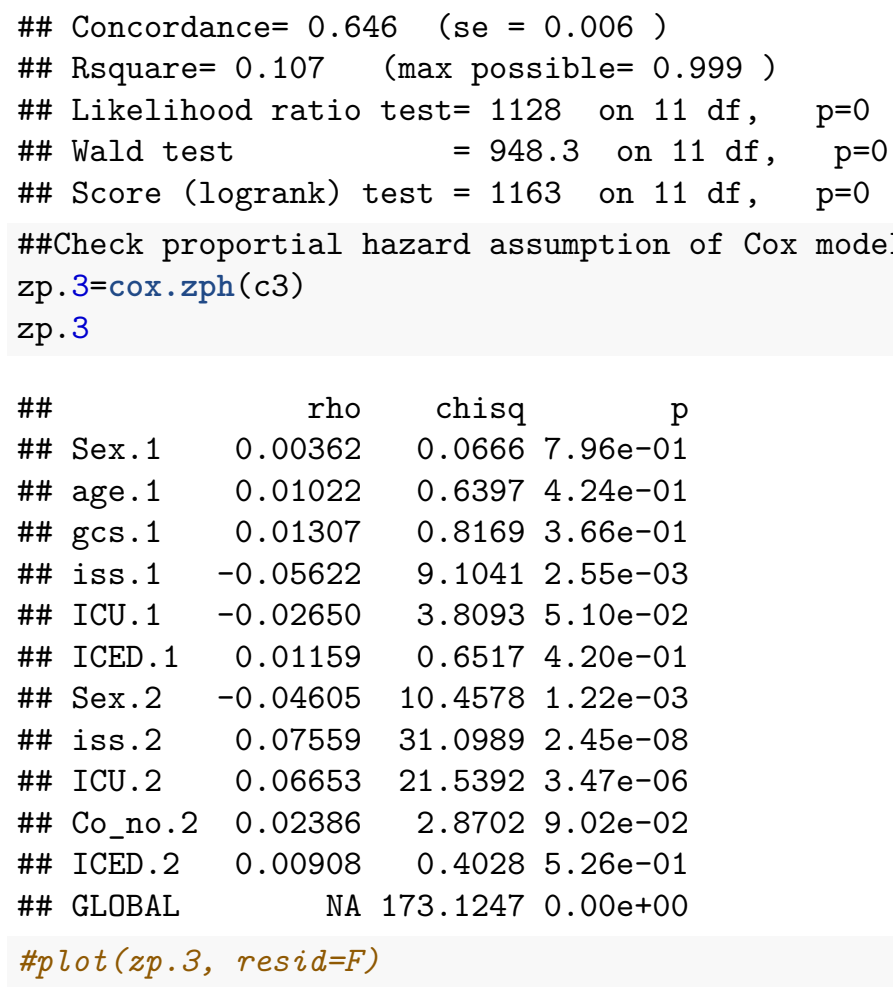

\section{Time to death outcome}

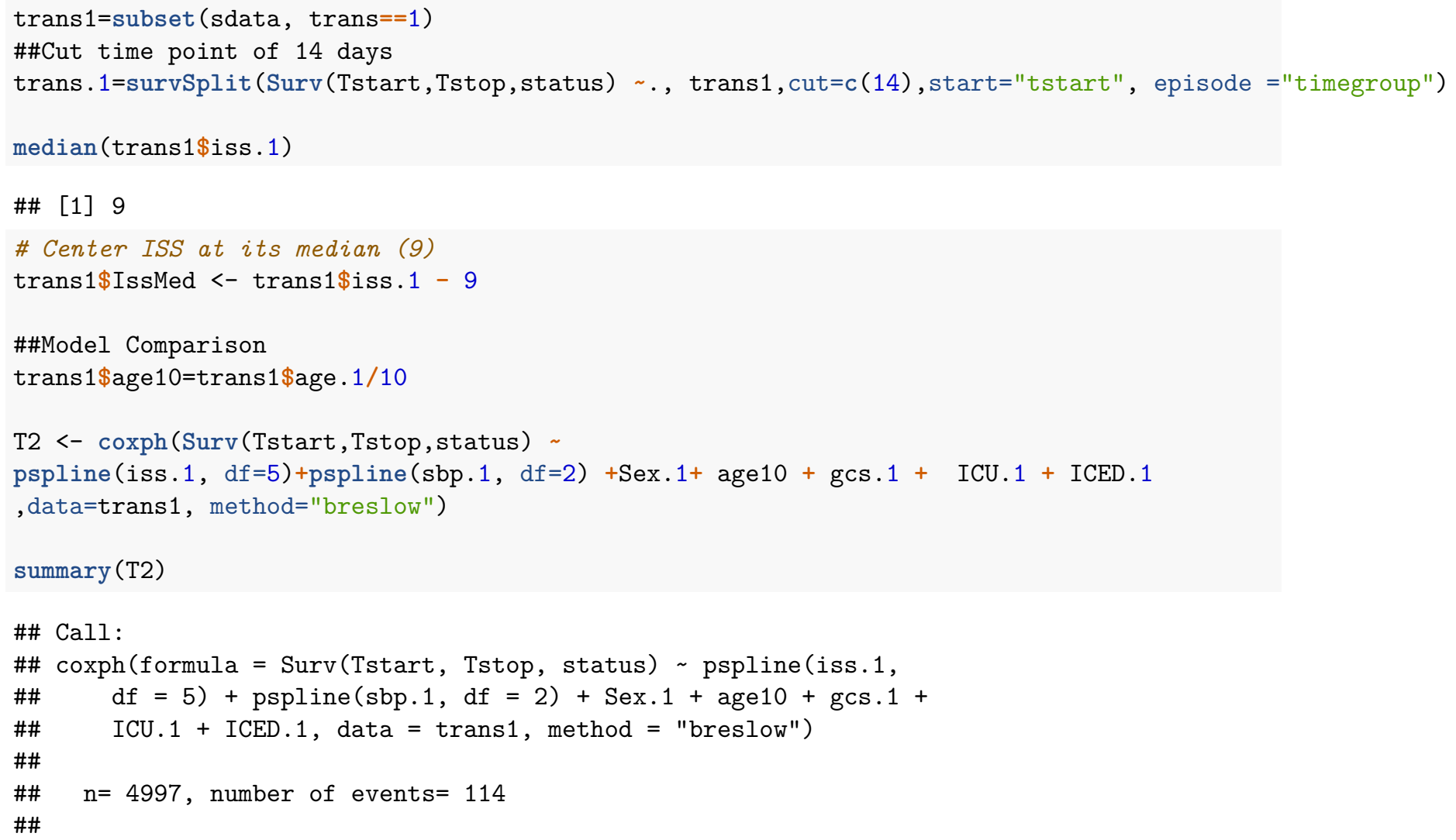




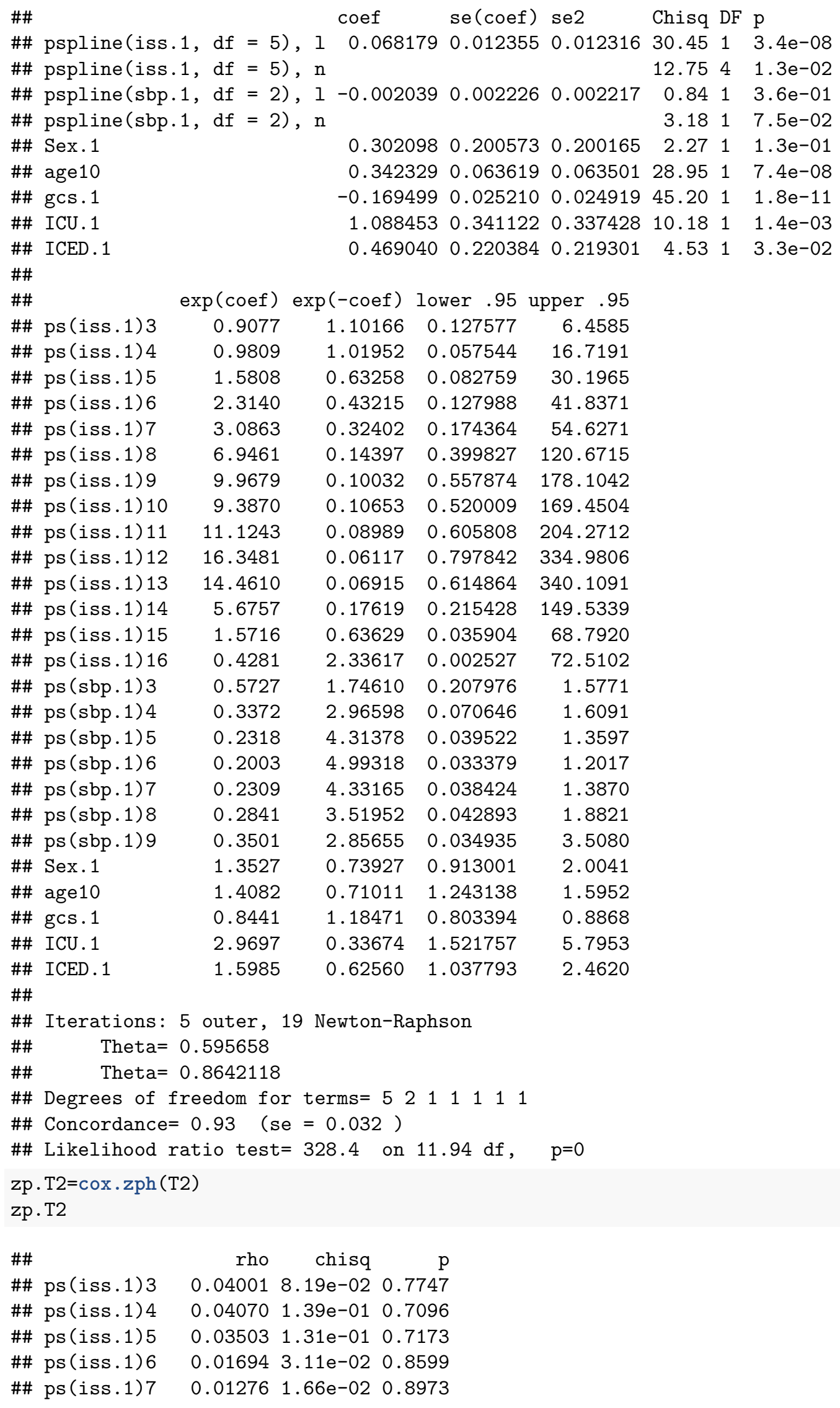




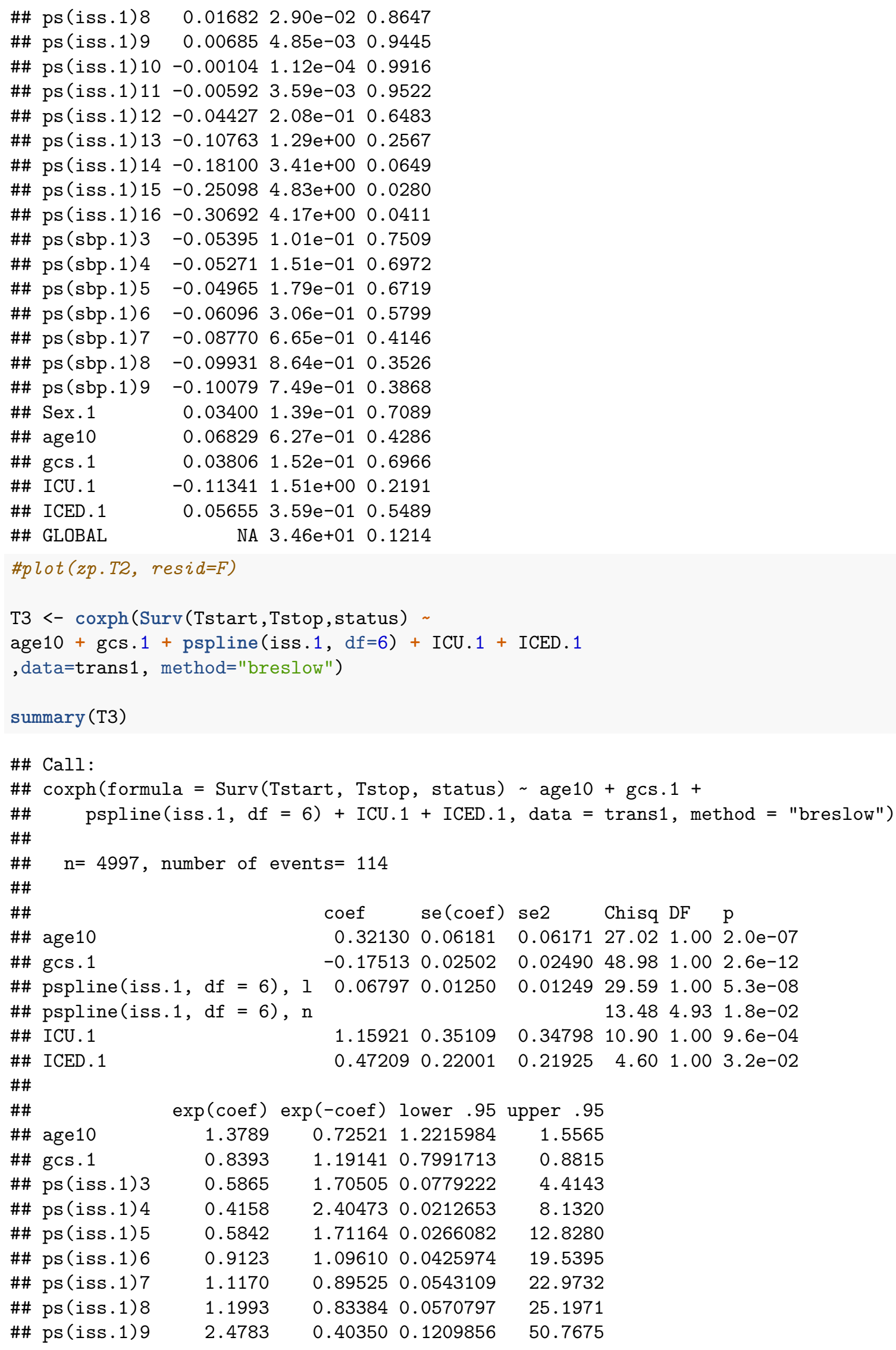

$\begin{array}{lllll}\text { \#\# age10 } & 1.3789 & 0.72521 & 1.2215984 & 1.5565\end{array}$

$\begin{array}{lllll}\text { \#\# gcs.1 } & 0.8393 & 1.19141 & 0.7991713 & 0.8815\end{array}$

$\begin{array}{lllll}\text { \#\# ps(iss.1)3 } & 0.5865 & 1.70505 & 0.0779222 & 4.4143\end{array}$

$\begin{array}{lllll}\text { \#\# ps(iss.1) } 4 & 0.4158 & 2.40473 & 0.0212653 & 8.1320\end{array}$

$\begin{array}{lllll}\text { \#\# ps(iss.1) } 5 & 0.5842 & 1.71164 & 0.0266082 & 12.8280\end{array}$

$\begin{array}{lllll}\text { \#\# ps(iss.1) } 6 & 0.9123 & 1.09610 & 0.0425974 & 19.5395\end{array}$

$\begin{array}{lllll}\text { \#\# ps(iss.1)7 } & 1.1170 & 0.89525 & 0.0543109 & 22.9732\end{array}$

$\begin{array}{lllll}\text { \#\# ps(iss.1)8 } & 1.1993 & 0.83384 & 0.0570797 & 25.1971\end{array}$

$\begin{array}{lllll}\text { \#\# ps(iss.1) } 9 & 2.4783 & 0.40350 & 0.1209856 & 50.7675\end{array}$ 


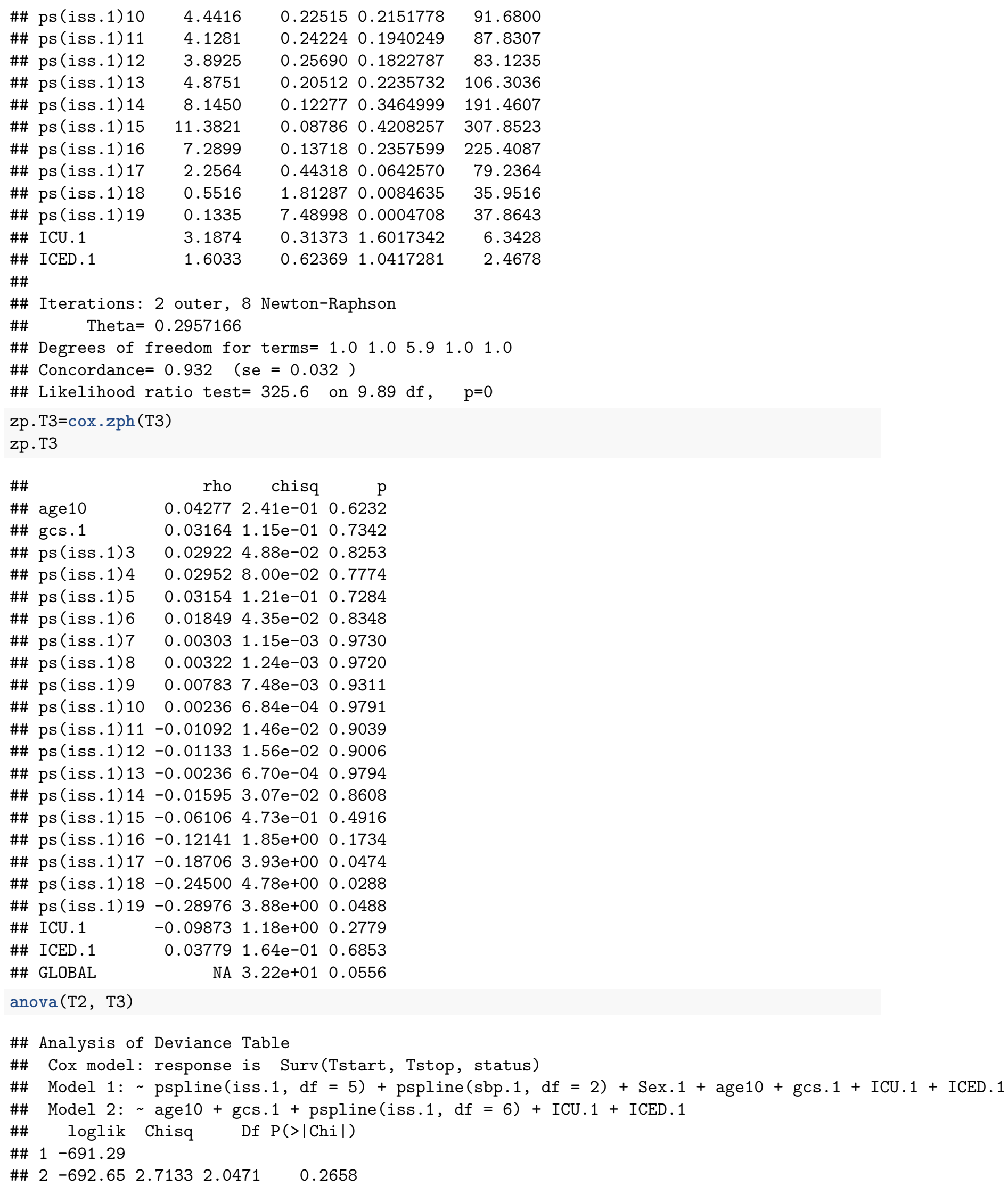




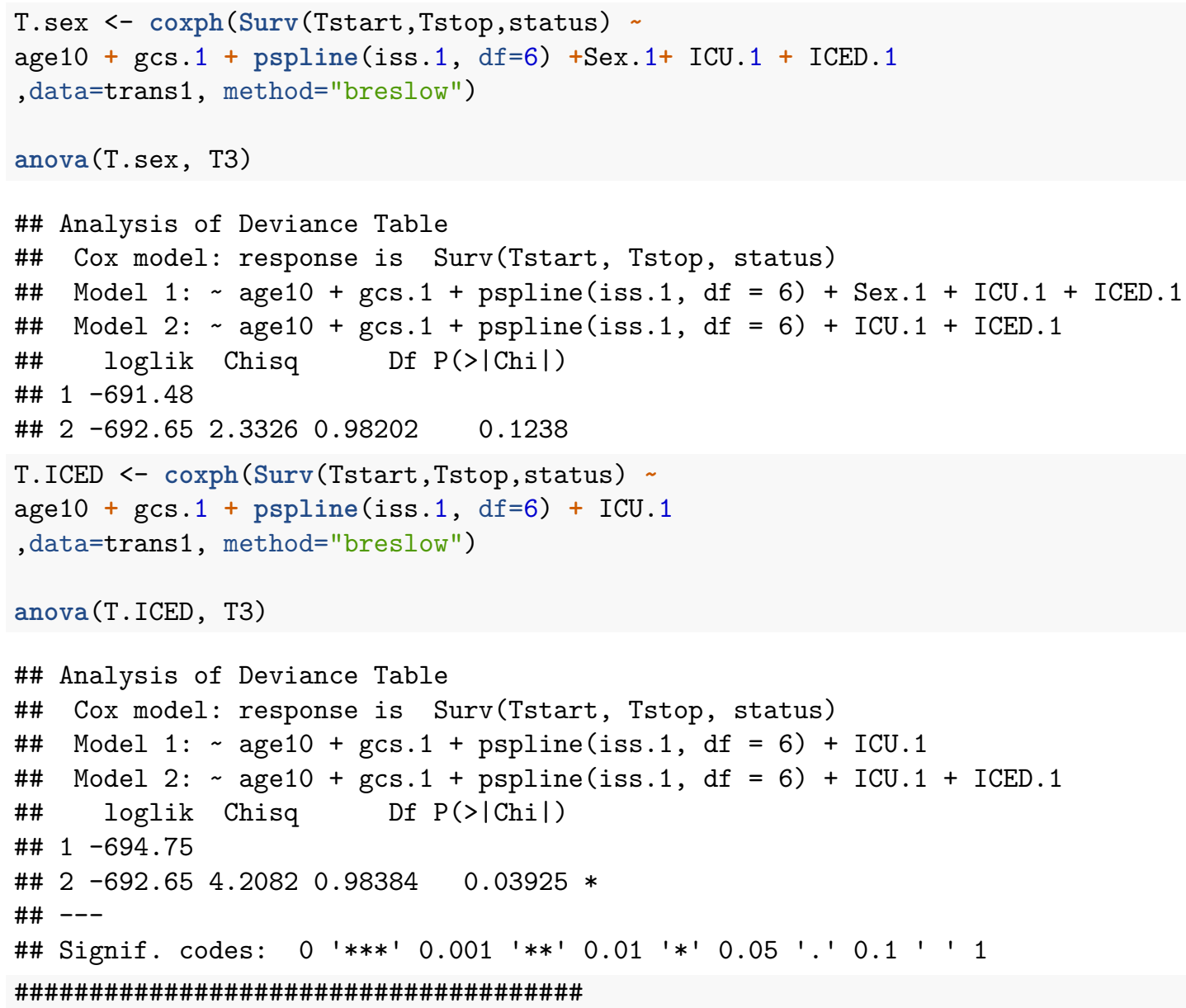

\section{Final model for time to death outcome (Table 3)}

\#\#T3 is our final model

\#Extract HR with $95 \%$ CI

T3. HR=round ( summary $(T 3) \$$ conf . int $[, c(1,3,4)]$, digits $=2$ )

T3. HR

$\begin{array}{lrrr}\text { \#\# } & \text { exp(coef) } & \text { lower .95 upper } .95 \\ \text { \#\# age10 } & 1.38 & 1.22 & 1.56 \\ \text { \#\# gcs.1 } & 0.84 & 0.80 & 0.88 \\ \text { \#\# ps(iss.1)3 } & 0.59 & 0.08 & 4.41 \\ \text { \#\# ps(iss.1)4 } & 0.42 & 0.02 & 8.13 \\ \text { \#\# ps(iss.1)5 } & 0.58 & 0.03 & 12.83 \\ \text { \#\# ps(iss.1)6 } & 0.91 & 0.04 & 19.54 \\ \text { \#\# ps(iss.1)7 } & 1.12 & 0.05 & 22.97 \\ \text { \#\# ps(iss.1)8 } & 1.20 & 0.06 & 25.20 \\ \text { \#\# ps(iss.1)9 } & 2.48 & 0.12 & 50.77 \\ \text { \#\# ps(iss.1)10 } & 4.44 & 0.22 & 91.68 \\ \text { \#\# ps(iss.1)11 } & 4.13 & 0.19 & 87.83 \\ \text { \#\# ps(iss.1)12 } & 3.89 & 0.18 & 83.12 \\ \text { \#\# ps(iss.1)13 } & 4.88 & 0.22 & 106.30 \\ \text { \#\# ps(iss.1)14 } & 8.15 & 0.35 & 191.46\end{array}$




$\begin{array}{lrrr}\text { \#\# ps(iss.1)15 } & 11.38 & 0.42 & 307.85 \\ \text { \#\# ps(iss.1)16 } & 7.29 & 0.24 & 225.41 \\ \text { \#\# ps(iss.1)17 } & 2.26 & 0.06 & 79.24 \\ \text { \#\# ps(iss.1)18 } & 0.55 & 0.01 & 35.95 \\ \text { \#\# ps(iss.1)19 } & 0.13 & 0.00 & 37.86 \\ \text { \#\# ICU.1 } & 3.19 & 1.60 & 6.34 \\ \text { \#\# ICED.1 } & 1.60 & 1.04 & 2.47\end{array}$

\#Extract $p$-value

T3.P=round ( $\operatorname{summary}(\mathrm{T} 3) \$ \operatorname{coefficient}[, 6]$, digits $=3$ )

T3.P

$\begin{array}{lrr}\# \# & \text { age10 } & \text { gcs.1 } \\ \# \# & 0.000 & 0.000 \\ \# \# \text { pspline(iss.1, df }= & 6 \text { ), } 1 \text { pspline(iss.1, df }=6 \text { ), n } \\ \text { \#\# } & 0.000 & 0.018 \\ \# \# & \text { ICU.1 } & \text { ICED.1 } \\ \# \# & 0.001 & 0.032\end{array}$

\section{Time to discharge outcome}

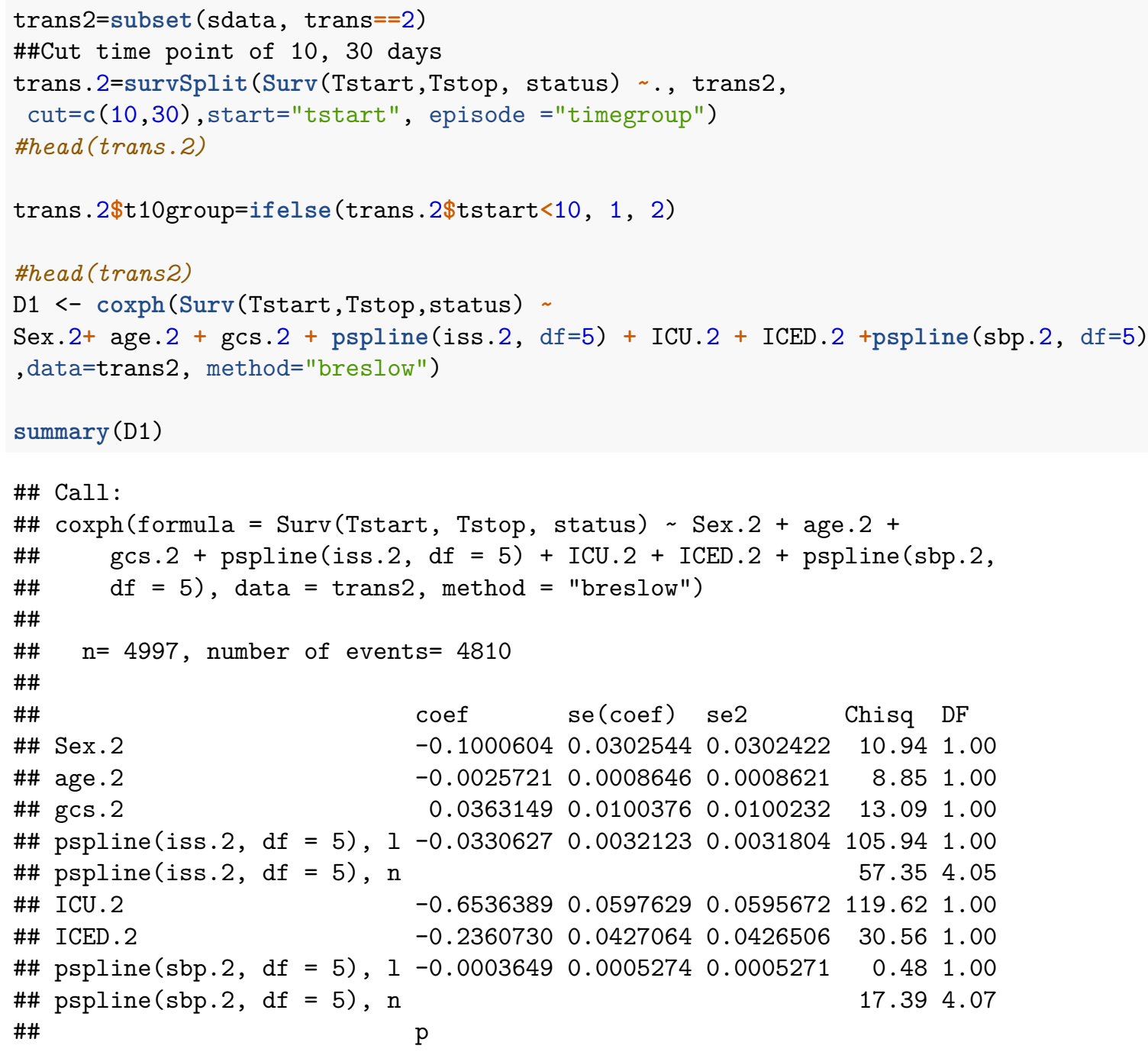




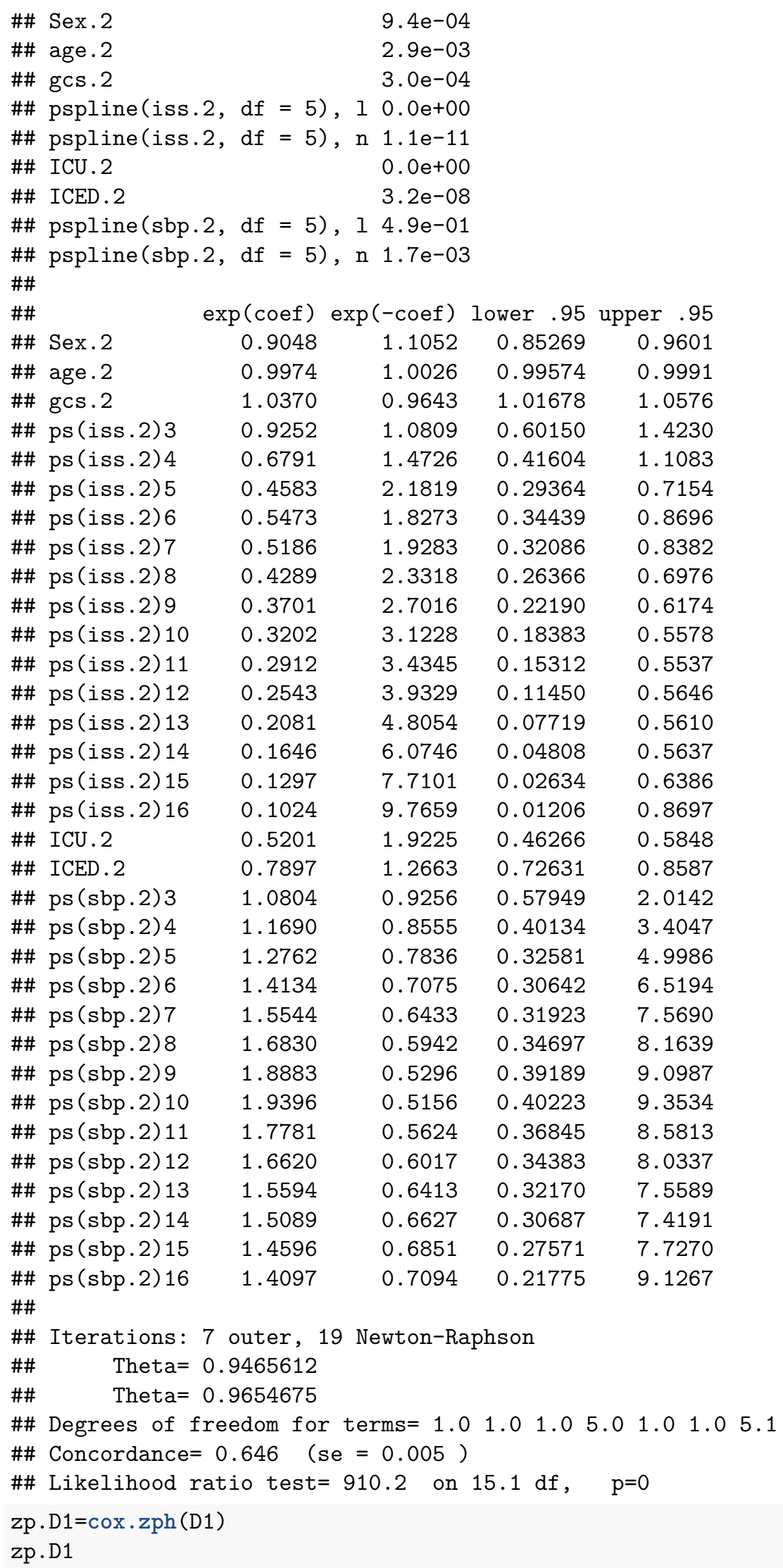




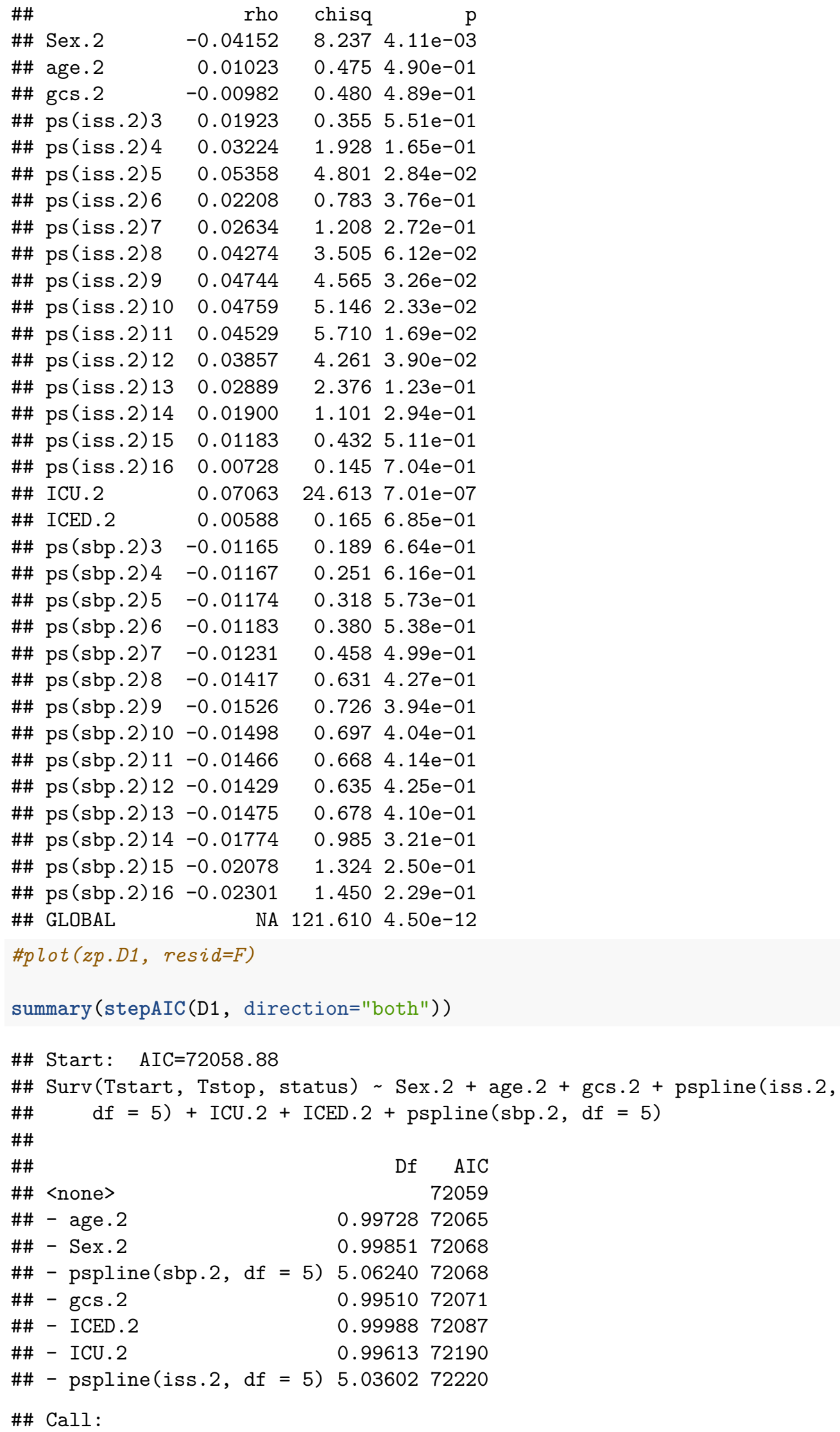




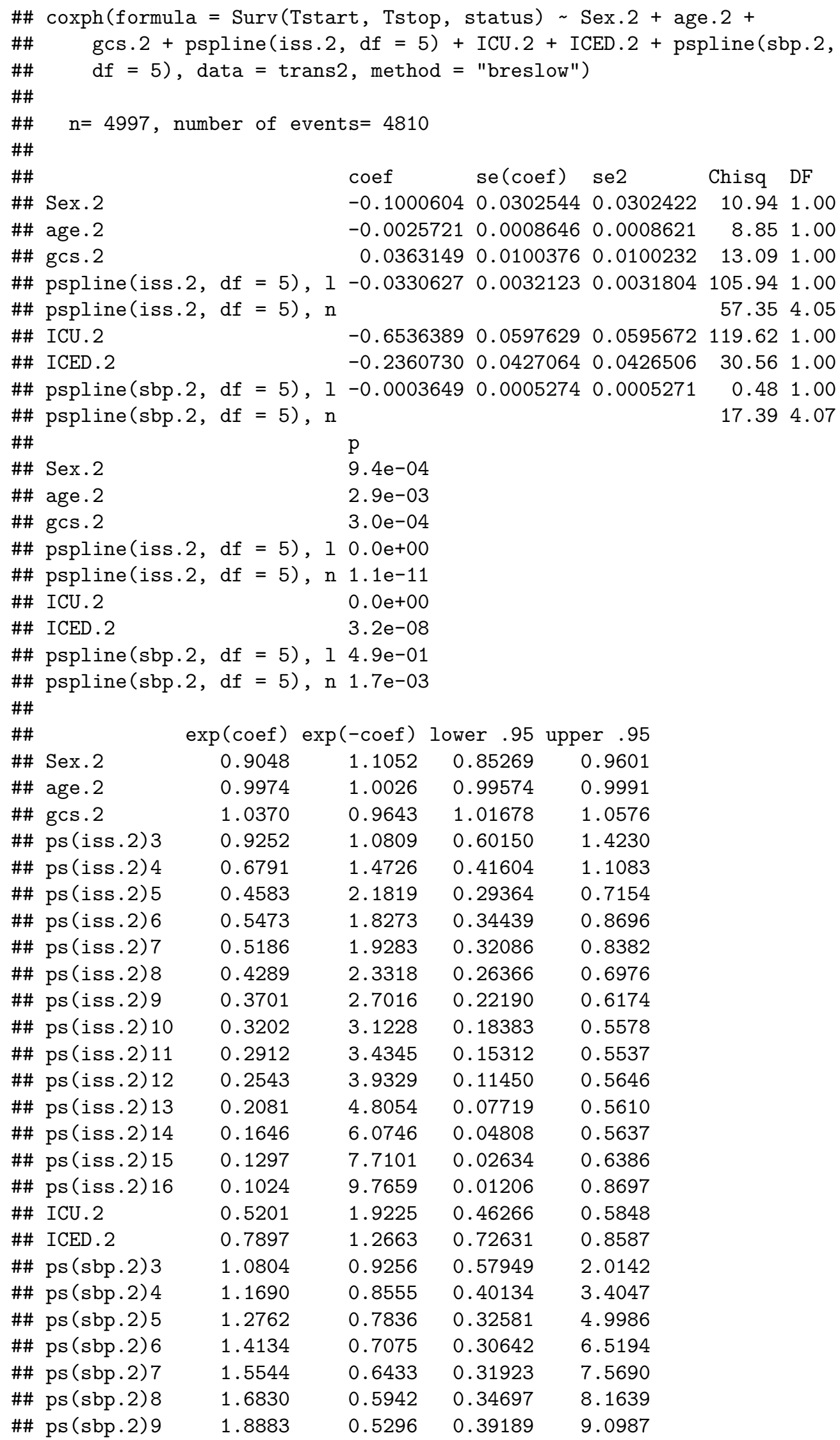




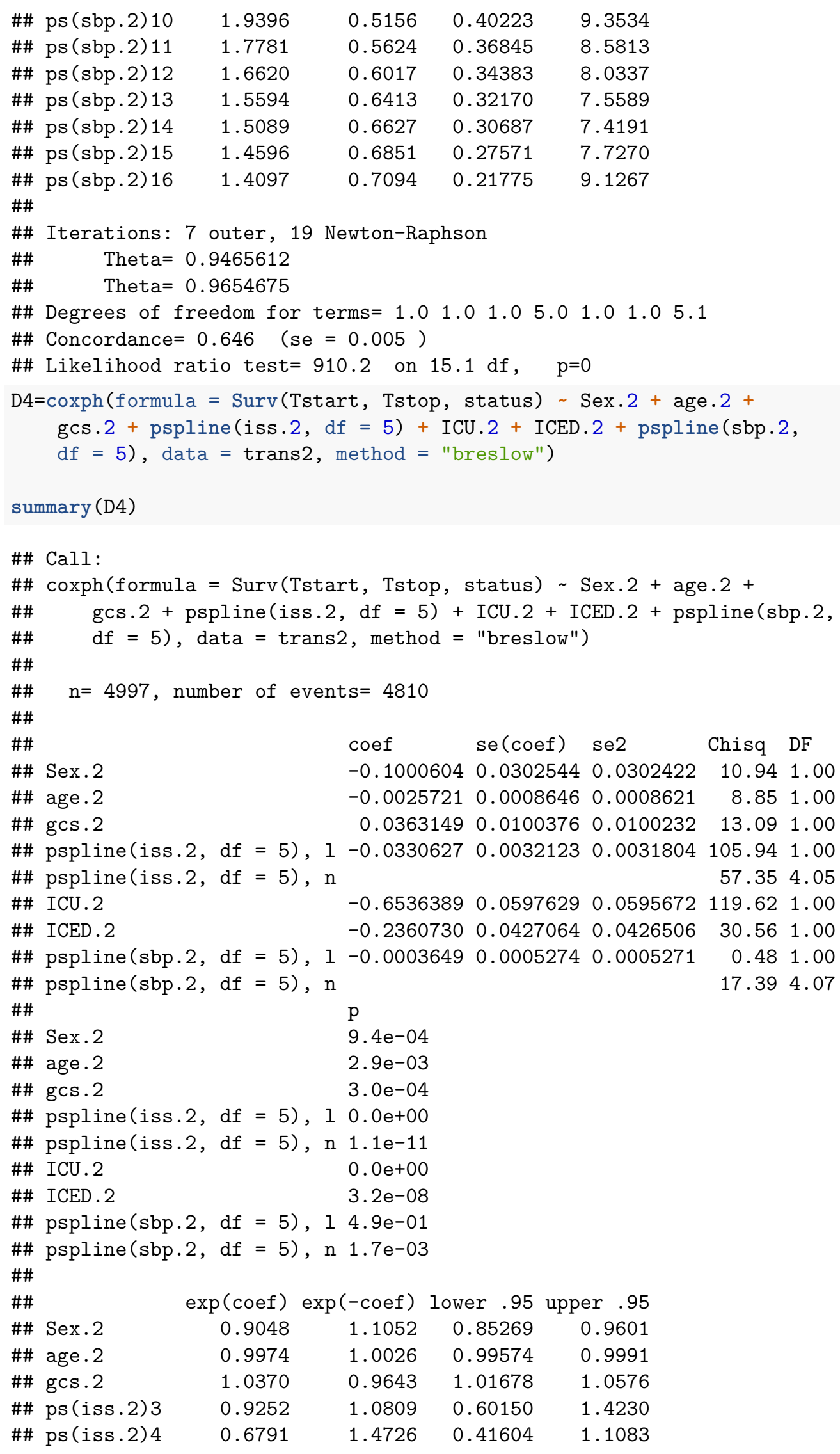




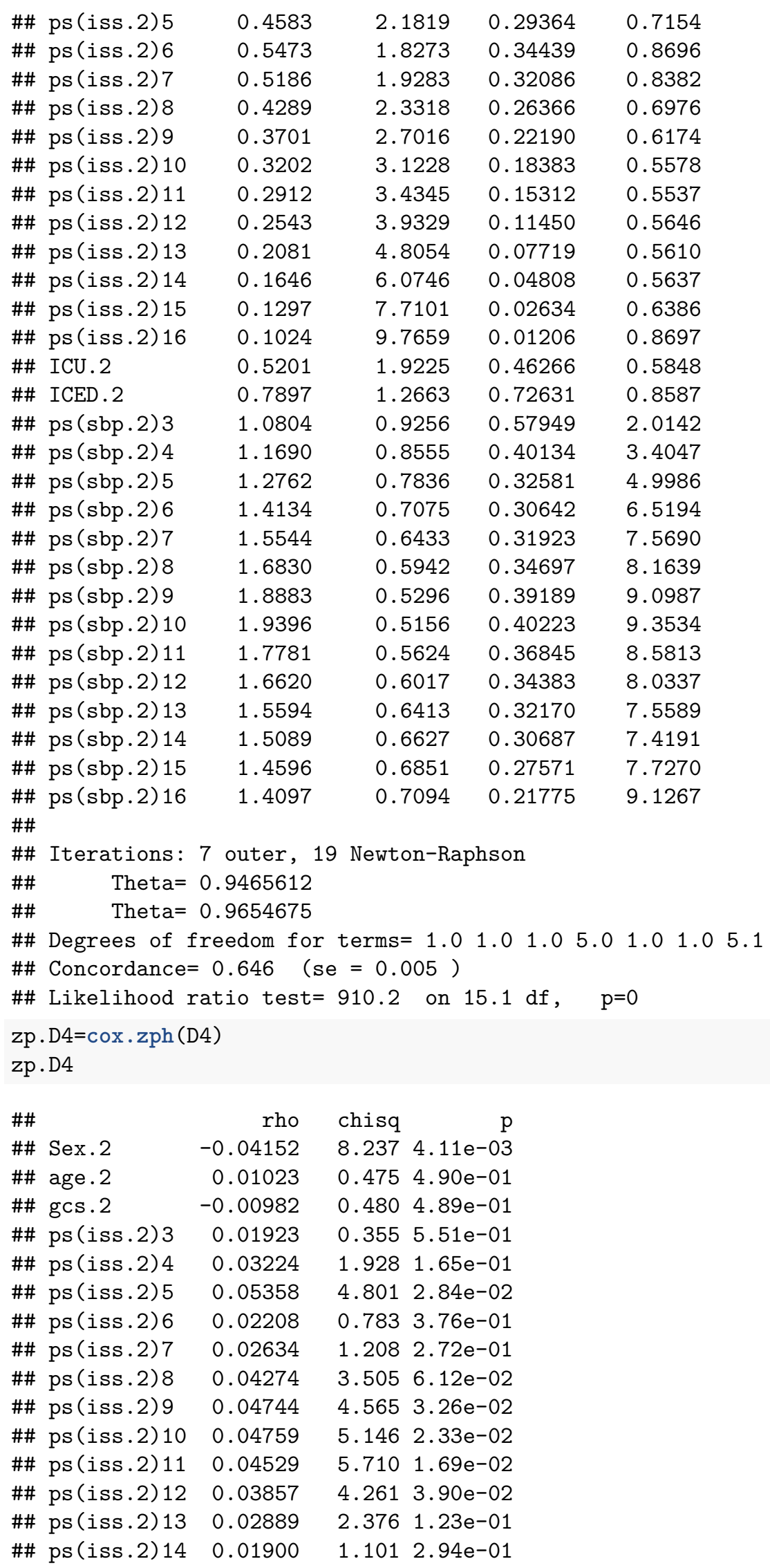




\begin{tabular}{|c|c|c|c|c|}
\hline & ps (1s & & & \\
\hline \# & ps & 728 & & \\
\hline & ICU. 2 & 7063 & & -07 \\
\hline & CED . & & & \\
\hline & $s(s$ & 35 & & 01 \\
\hline & 2) 4 & 167 & 0.251 & -01 \\
\hline & - & 74 & & \\
\hline & 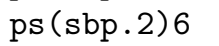 & 3 & & \\
\hline & 7 & 1 & & -01 \\
\hline & $s$ & -0 & 0. & -01 \\
\hline & 9 & -0 . & & -01 \\
\hline & & & & \\
\hline & (sbp.2 & -0 & 0. & $4.14 \mathrm{e}-01$ \\
\hline & 2 & -0 & & $4.25 e-01$ \\
\hline & & -0 . & & -01 \\
\hline & & -0 & & $3.21 e-01$ \\
\hline & & -0 & 1. & $2.50 e-01$ \\
\hline & . 2) 1 & -0.02301 & 1.45 & $2.29 e-01$ \\
\hline & & & 1.61 & $4.50 e-12$ \\
\hline
\end{tabular}

\#\#\#\#\#\#\#\#\#\#\#\#\#\#\#\#\#\#\#\#\#\#\#\#\#\#\#\#\#\#\#\#\#

D2 <- coxph(Surv(tstart, Tstop, status)

Sex.2:strata(t10group)+ age.2 + gcs.2 + pspline(iss.2, df=6) + ICU.2:strata(timegroup) + ICED.2 +psplin , data=trans.2, method="breslow")

summary (D2)

\#\# Call:

\#\# coxph(formula $=\operatorname{Surv(tstart,~Tstop,~status)~\sim ~Sex.2:strata(t10group)~+~}$

\#\# age.2 + gcs.2 + pspline(iss.2, df =6) + ICU.2:strata(timegroup) +

\#\# ICED.2 + pspline (sbp.2, df =5), data = trans.2, method = "breslow")

\#\#

\#\# $\mathrm{n}=6823$, number of events $=4810$

\#\#

\#\# coef se(coef) se2 $\quad$ Chisq DF

\#\# age.2 $\quad-0.00241380 .00086950 .0008660 \quad 7.711 .00$

$\begin{array}{lllllll}\text { \#\# gcs.2 } & 0.0364511 & 0.0100545 & 0.0100359 & 13.14 & 1.00\end{array}$

\#\# pspline(iss.2, df =6), 1 -0.0335370 $0.0032156 \quad 0.0031939108 .781 .00$

\#\# pspline(iss.2, df =6), $\mathrm{n} \quad 56.765 .06$

\#\# ICED.2 $\quad-0.2344071 \quad 0.04280850 .0427521 \quad 29.981 .00$

\#\# pspline (sbp.2, df = 5), $1-0.00036840 .00052750 .0005272 \quad 0.491 .00$

\#\# pspline (sbp.2, df $=5), \mathrm{n}$

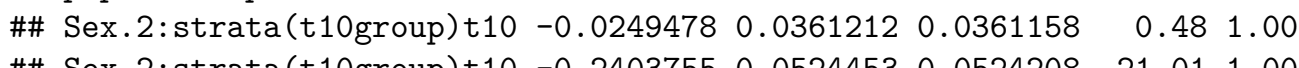

\#\# Sex.2:strata(t10group)t10 $\quad-0.2403755 \quad 0.0524453 \quad 0.0524208 \quad 21.011 .00$

\#\# ICU.2:strata(timegroup)ti $-0.9468894 \quad 0.0835755 \quad 0.0834834 \quad 128.361 .00$

\#\# ICU.2:strata(timegroup)ti $-0.4984600 \quad 0.0793111 \quad 0.0791563 \quad 39.501 .00$

$\begin{array}{lllllll}\text { \# ICU.2:strata(timegroup)ti } & -0.1168711 & 0.1474939 & 0.1472307 & 0.63 & 1.00\end{array}$

\#\#

\#\# age. 2

\#\# gcs. $2.9 e-04$

\#\# pspline (iss.2, df =6), $10.0 e+00$

\#\# pspline (iss.2, df =6), n 6.1e-11

\#\# ICED. $2 \quad 4.4 \mathrm{e}-08$

\#\# pspline (sbp.2, df $=5), 14.9 \mathrm{e}-01$

\#\# pspline (sbp.2, df =5), n 9.4e-04 


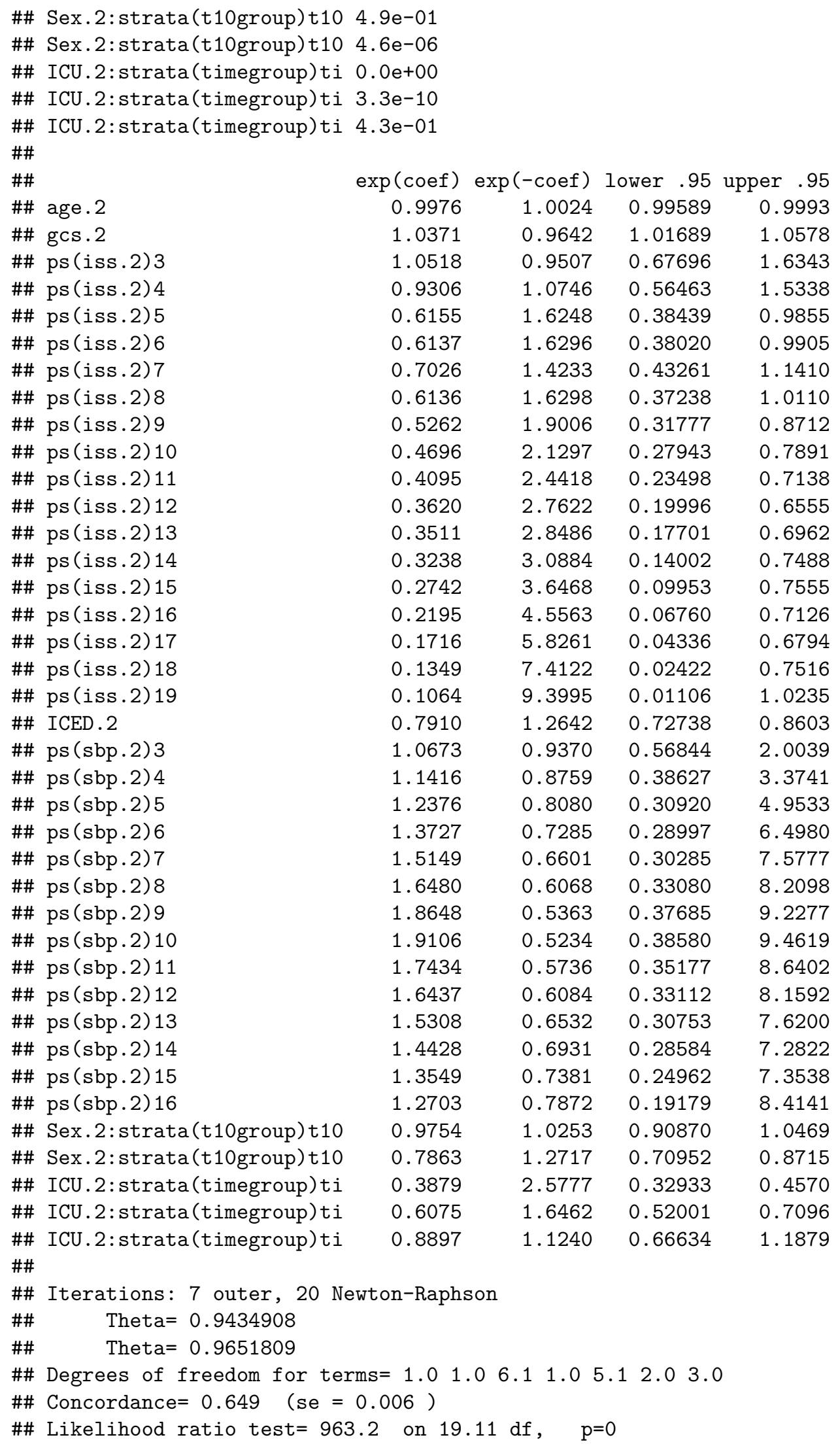


$\mathrm{zp} \cdot \mathrm{D} 2=\operatorname{cox} \cdot \mathrm{zph}(\mathrm{D} 2)$

zp.D2

\begin{tabular}{|c|c|c|c|c|}
\hline \#\# & & rho & chisq & $\mathrm{p}$ \\
\hline & age. 2 & 0.00831 & 0.3146 & 0.574875 \\
\hline \#\# & $\operatorname{gcs} .2$ & -0.01224 & 0.7465 & 0.387585 \\
\hline \#\# & ps (iss.2) 3 & 0.00918 & 0.0847 & 0.771072 \\
\hline \#\# & ps (iss.2) 4 & 0.01639 & 0.6045 & 0.436884 \\
\hline \#\# & ps (iss.2) 5 & 0.03637 & 2.7069 & 0.099917 \\
\hline \#\# & ps (iss.2) 6 & 0.03282 & 1.9471 & 0.162904 \\
\hline \#\# & ps (iss. 2) 7 & 0.00268 & 0.0147 & 0.903501 \\
\hline \#\# & ps (iss.2) 8 & 0.01970 & 0.8108 & 0.367888 \\
\hline \#\# & ps (iss.2) 9 & 0.03411 & 2.5181 & 0.112550 \\
\hline \#\# & ps (iss. 2) 10 & 0.03404 & 2.6211 & 0.105449 \\
\hline \#\# & ps (iss. 2) 11 & 0.03289 & 2.4836 & 0.115038 \\
\hline \#\# & ps (iss. 2) 12 & 0.03503 & 3.1701 & 0.074997 \\
\hline \#\# & ps (iss. 2) 13 & 0.03795 & 4.5119 & 0.033659 \\
\hline \#\# & ps (iss. 2) 14 & 0.03577 & 3.9633 & 0.046501 \\
\hline \#\# & ps (iss. 2) 15 & 0.03008 & 2.5235 & 0.112165 \\
\hline \#\# & ps (iss. 2) 16 & 0.02186 & 1.3574 & 0.243988 \\
\hline \#\# & ps (iss. 2) 17 & 0.01370 & 0.6065 & 0.436126 \\
\hline \#\# & ps (iss. 2) 18 & 0.00805 & 0.2204 & 0.638724 \\
\hline \#\# & ps (iss. 2) 19 & 0.00445 & 0.0605 & 0.805653 \\
\hline \#\# & ICED . 2 & 0.00366 & 0.0636 & 0.800910 \\
\hline \#\# & $\mathrm{ps}(\mathrm{sbp} .2) 3$ & -0.01345 & 0.2650 & 0.606679 \\
\hline \#\# & ps (sbp. 2) 4 & -0.01345 & 0.3507 & 0.553734 \\
\hline \#\# & $\mathrm{ps}(\mathrm{sbp} .2) 5$ & -0.01347 & 0.4377 & 0.508211 \\
\hline \#\# & $\mathrm{ps}(\mathrm{sbp} .2) 6$ & -0.01346 & 0.5106 & 0.474897 \\
\hline \#\# & $\mathrm{ps}(\mathrm{sbp} .2) 7$ & -0.01389 & 0.6025 & 0.437613 \\
\hline \#\# & $\mathrm{ps}(\mathrm{sbp} .2) 8$ & -0.01567 & 0.7972 & 0.371932 \\
\hline \#\# & ps (sbp.2) 9 & -0.01656 & 0.8826 & 0.347497 \\
\hline \#\# & ps (sbp. 2) 10 & -0.01632 & 0.8542 & 0.355373 \\
\hline \#\# & $\mathrm{ps}(\mathrm{sbp} .2) 11$ & -0.01603 & 0.8256 & 0.363541 \\
\hline \#\# & $\mathrm{ps}(\mathrm{sbp} .2) 12$ & -0.01548 & 0.7698 & 0.380285 \\
\hline \#\# & ps (sbp. 2) 13 & -0.01603 & 0.8258 & 0.363502 \\
\hline \#\# & $\mathrm{ps}(\mathrm{sbp} .2) 14$ & -0.01920 & 1.1905 & 0.275235 \\
\hline \#\# & $\mathrm{ps}(\mathrm{sbp} .2) 15$ & -0.02242 & 1.5875 & 0.207689 \\
\hline \#\# & $\mathrm{ps}(\mathrm{sbp} .2) 16$ & -0.02484 & 1.7359 & 0.187664 \\
\hline \#\# & Sex.2: strata(t10group)t10group=1 & -0.00659 & 0.2087 & 0.647766 \\
\hline \#\# & Sex.2: strata(t10group)t10group $=2$ & 0.00930 & 0.4076 & 0.523179 \\
\hline \#\# & ICU. 2: strata (timegroup) timegroup=1 & 0.02701 & 3.8324 & 0.050271 \\
\hline \#\# & ICU. 2 : strata (timegroup) timegroup=2 & 0.00653 & 0.2003 & 0.654474 \\
\hline \#\# & ICU. 2 : strata (timegroup) timegroup=3 & -0.00173 & 0.0138 & 0.906617 \\
\hline \#\# & GLOBAL & NA & 78.3039 & 0.000191 \\
\hline
\end{tabular}

$\# p \operatorname{lot}(z p . D 2$, resid=F)

trans. 2\$age10=trans . 2\$age $.2 / 10$

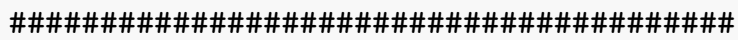

D3 <- coxph(Surv(tstart, Tstop, status)

pspline(iss.2, df=7) +pspline(sbp.2, df=5)+Sex.2:strata(t10group)+ age10 + gcs.2 + ICU.2:strata(timegr , data=trans.2, method="breslow")

summary (D3) 


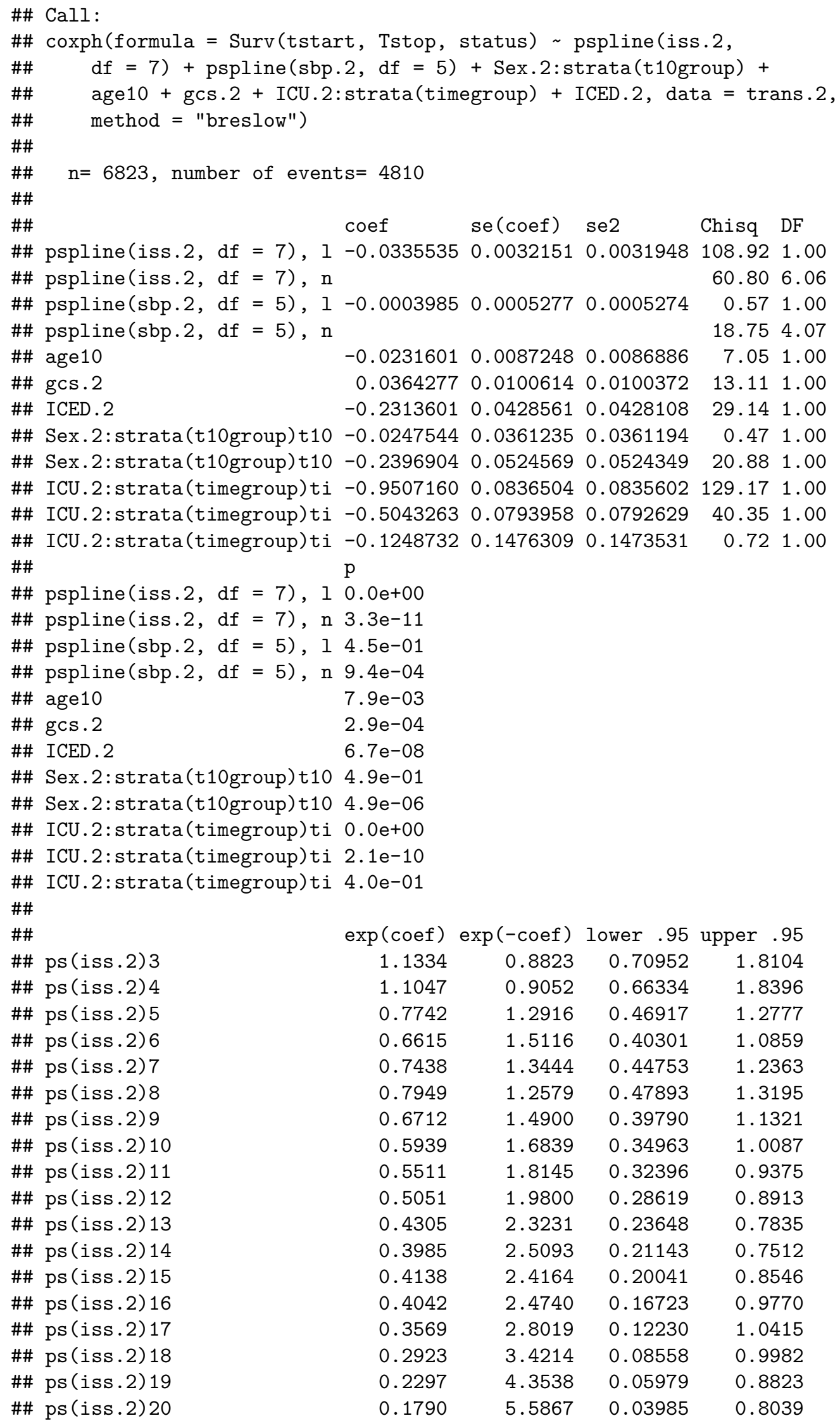

$\begin{array}{rrrr}\exp (\text { coef }) & \text { exp (-coef) } & \text { lower .95 } & \text { upper.95 } \\ 1.1334 & 0.8823 & 0.70952 & 1.8104 \\ 1.1047 & 0.9052 & 0.66334 & 1.8396 \\ 0.7742 & 1.2916 & 0.46917 & 1.2777 \\ 0.6615 & 1.5116 & 0.40301 & 1.0859 \\ 0.7438 & 1.3444 & 0.44753 & 1.2363 \\ 0.7949 & 1.2579 & 0.47893 & 1.3195 \\ 0.6712 & 1.4900 & 0.39790 & 1.1321 \\ 0.5939 & 1.6839 & 0.34963 & 1.0087 \\ 0.5511 & 1.8145 & 0.32396 & 0.9375 \\ 0.5051 & 1.9800 & 0.28619 & 0.8913 \\ 0.4305 & 2.3231 & 0.23648 & 0.7835 \\ 0.3985 & 2.5093 & 0.21143 & 0.7512 \\ 0.4138 & 2.4164 & 0.20041 & 0.8546 \\ 0.4042 & 2.4740 & 0.16723 & 0.9770 \\ 0.3569 & 2.8019 & 0.12230 & 1.0415 \\ 0.2923 & 3.4214 & 0.08558 & 0.9982 \\ 0.2297 & 4.3538 & 0.05979 & 0.8823 \\ 0.1790 & 5.5867 & 0.03985 & 0.8039\end{array}$




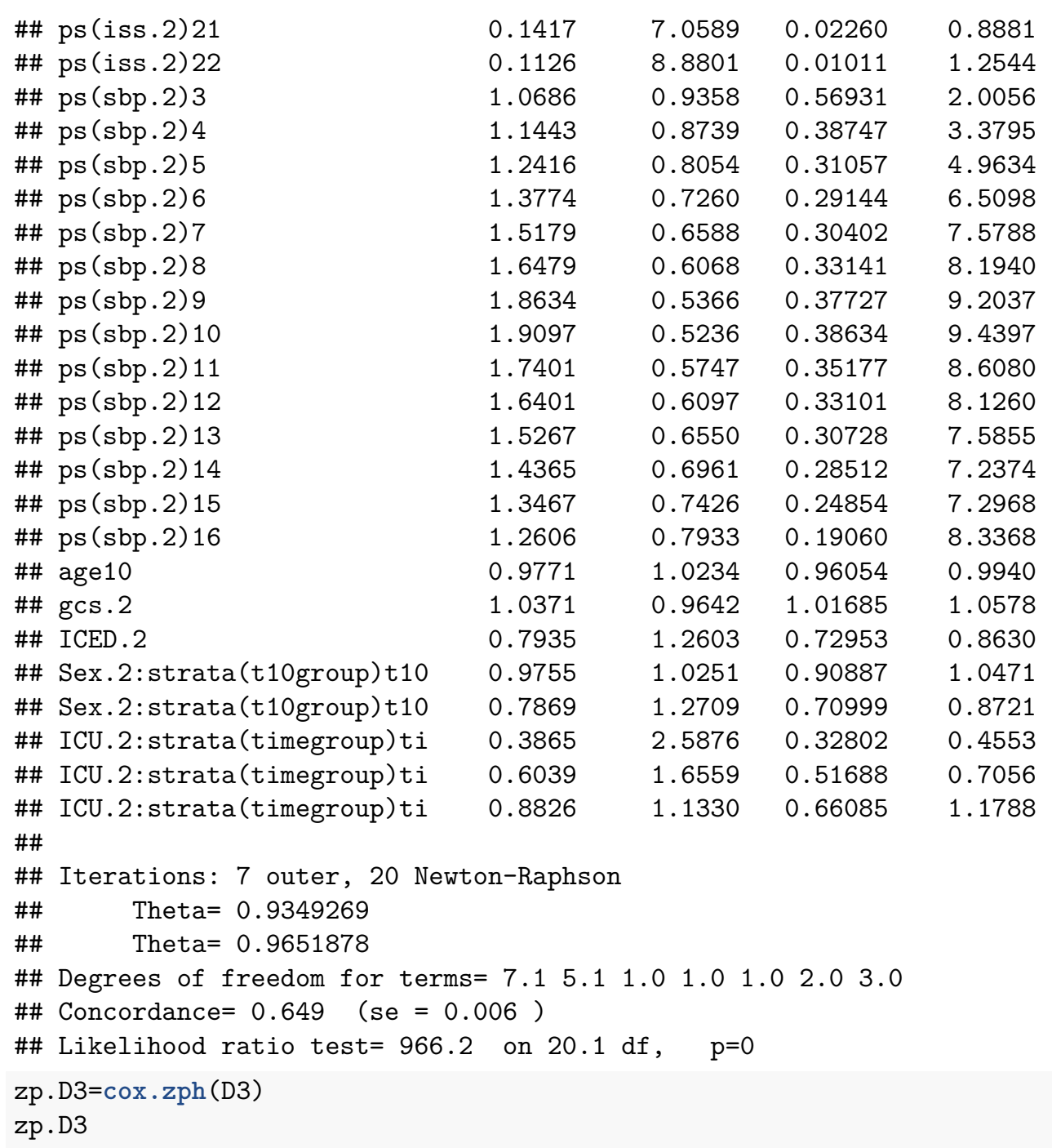

$\begin{array}{llll}0.1417 & 7.0589 & 0.02260 & 0.8881 \\ 0.1126 & 8.8801 & 0.01011 & 1.2544 \\ 1.0686 & 0.9358 & 0.56931 & 2.0056 \\ 1.1443 & 0.8739 & 0.38747 & 3.3795 \\ 1.2416 & 0.8054 & 0.31057 & 4.9634 \\ 1.3774 & 0.7260 & 0.29144 & 6.5098 \\ 1.5179 & 0.6588 & 0.30402 & 7.5788 \\ 1.6479 & 0.6068 & 0.33141 & 8.1940 \\ 1.8634 & 0.5366 & 0.37727 & 9.2037 \\ 1.9097 & 0.5236 & 0.38634 & 9.4397 \\ 1.7401 & 0.5747 & 0.35177 & 8.6080 \\ 1.6401 & 0.6097 & 0.33101 & 8.1260 \\ 1.5267 & 0.6550 & 0.30728 & 7.5855 \\ 1.4365 & 0.6961 & 0.28512 & 7.2374 \\ 1.3467 & 0.7426 & 0.24854 & 7.2968 \\ 1.2606 & 0.7933 & 0.19060 & 8.3368 \\ 0.9771 & 1.0234 & 0.96054 & 0.9940 \\ 1.0371 & 0.9642 & 1.01685 & 1.0578 \\ 0.7935 & 1.2603 & 0.72953 & 0.8630 \\ 0.9755 & 1.0251 & 0.90887 & 1.0471 \\ 0.7869 & 1.2709 & 0.70999 & 0.8721 \\ 0.3865 & 2.5876 & 0.32802 & 0.4553 \\ 0.6039 & 1.6559 & 0.51688 & 0.7056 \\ 0.8826 & 1.1330 & 0.66085 & 1.1788\end{array}$

\#\#

\#\# ps (iss. 2) 3

\#\# ps (iss. 2) 4

\#\# ps (iss. 2) 5

\#\# ps(iss.2) 6

\#\# ps (iss.2) 7

\#\# ps(iss.2) 8

\#\# ps(iss.2) 9

\#\# ps (iss.2) 10

\#\# ps (iss.2) 11

\#\# ps (iss.2) 12

\#\# ps (iss.2) 13

\#\# ps (iss.2) 14

\#\# ps (iss.2) 15

\#\# ps (iss.2) 16

\#\# ps (iss.2) 17

\#\# ps (iss.2) 18

\#\# ps (iss.2) 19

\#\# ps(iss.2) 20

\#\# ps (iss.2) 21 


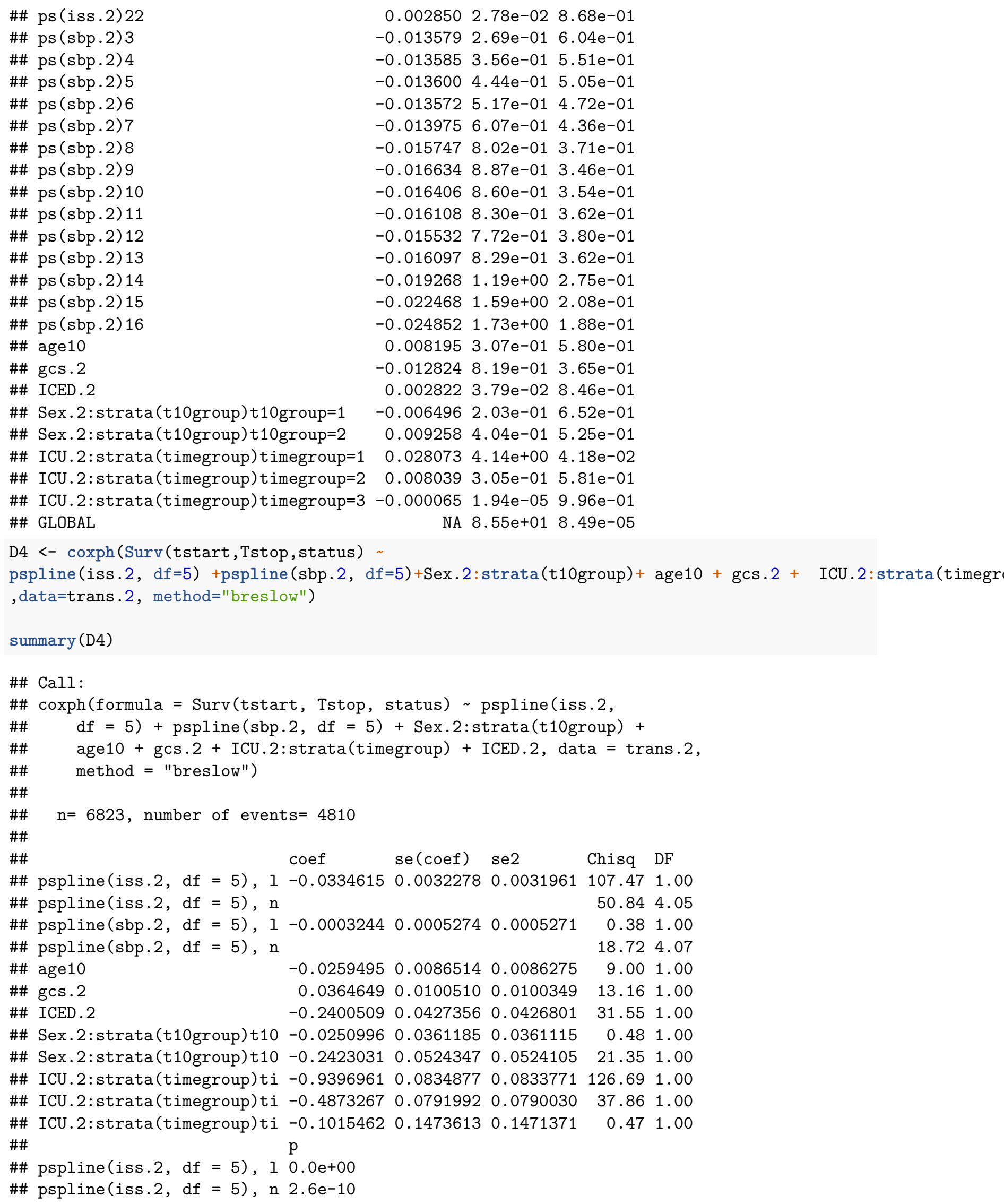




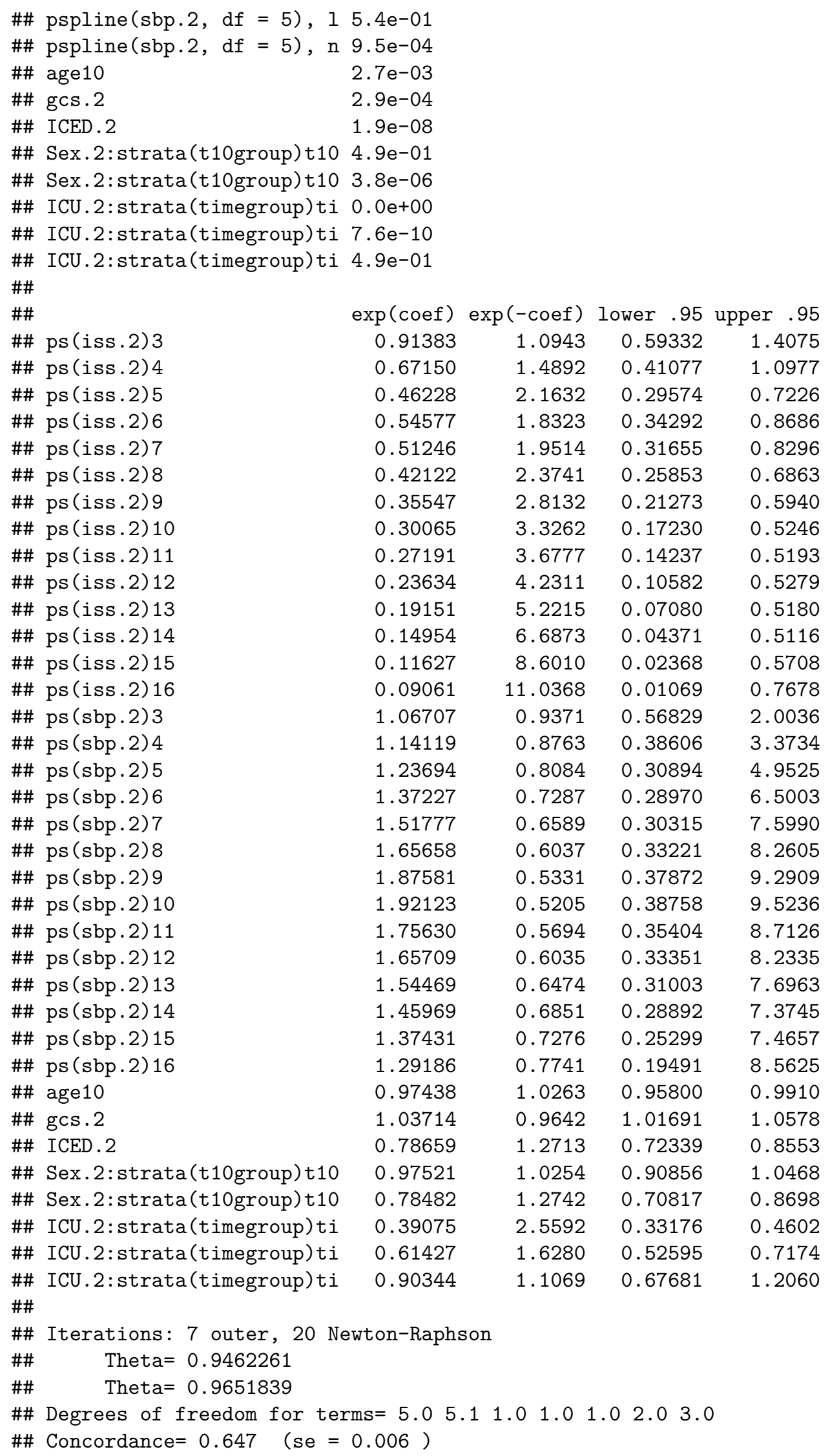


\#\# Likelihood ratio test= 956.1 on $18.09 \mathrm{df}, \quad \mathrm{p}=0$

$\mathrm{zp} \cdot \mathrm{D} 4=\operatorname{cox} \cdot \mathrm{zph}(\mathrm{D} 4)$

zp.D4

\#\#

\#\# ps (iss. 2) 3

\#\# ps(iss.2) 4

\#\# ps(iss.2) 5

\#\# ps(iss.2) 6

\#\# ps(iss.2) 7

\#\# ps(iss.2)8

\#\# ps(iss.2) 9

\#\# ps (iss. 2) 10

\#\# ps(iss.2) 11

\#\# ps(iss.2) 12

\#\# ps(iss.2)13

\#\# ps(iss.2) 14

\#\# ps (iss.2) 15

\#\# ps (iss.2) 16

\#\# ps(sbp.2) 3

\#\# ps (sbp. 2) 4

\#\# ps (sbp.2) 5

\#\# ps(sbp.2) 6

\#\# ps (sbp.2)7

\#\# ps(sbp.2)8

\#\# ps(sbp.2) 9

\#\# ps (sbp.2) 10

\#\# ps(sbp.2)11

\#\# ps (sbp.2) 12

\#\# ps (sbp. 2) 13

\#\# ps (sbp. 2) 14

\#\# ps (sbp. 2) 15

\#\# ps (sbp.2) 16

\#\# age10

\#\# gcs.2

\#\# ICED. 2

\#\# Sex.2:strata(t10group)t10group=1

\#\# Sex.2:strata(t10group)t10group=2

\#\# ICU.2:strata(timegroup)timegroup=1

\#\# ICU.2:strata(timegroup)timegroup=2

\#\# ICU . 2:strata (timegroup)timegroup=3 -0.00

\#\# GLOBAL

anova (D3, D4) rho chisq $\mathrm{p}$

$\begin{array}{llll}0.01693 & 0.2764 & 0.599041\end{array}$

$0.03033 \quad 1.7135 \quad 0.190533$

$0.05260 \quad 4.6486 \quad 0.031078$

$0.02061 \quad 0.6868 \quad 0.407266$

$\begin{array}{llll}0.02499 & 1.0944 & 0.295502\end{array}$

$0.04147 \quad 3.3170 \quad 0.068565$

$\begin{array}{llll}0.04575 & 4.2523 & 0.039198\end{array}$

$\begin{array}{llll}0.04623 & 4.8669 & 0.027377\end{array}$

$0.04493 \quad 5.6886 \quad 0.017076$

$\begin{array}{llll}0.03893 & 4.4017 & 0.035903\end{array}$

$\begin{array}{llll}0.02964 & 2.5245 & 0.112092\end{array}$

$\begin{array}{llll}0.01982 & 1.2002 & 0.273287\end{array}$

$\begin{array}{llll}0.01255 & 0.4845 & 0.486408\end{array}$

$\begin{array}{llll}0.00789 & 0.1692 & 0.680856\end{array}$

$\begin{array}{llll}-0.01319 & 0.2558 & 0.613016\end{array}$

$\begin{array}{llll}-0.01320 & 0.3384 & 0.560769\end{array}$

$\begin{array}{llll}-0.01321 & 0.4221 & 0.515902\end{array}$

$\begin{array}{llll}-0.01319 & 0.4918 & 0.483145\end{array}$

$\begin{array}{llll}-0.01363 & 0.5814 & 0.445766\end{array}$

$\begin{array}{llll}-0.01541 & 0.7734 & 0.379174\end{array}$

$\begin{array}{llll}-0.01631 & 0.8593 & 0.353932\end{array}$

$\begin{array}{llll}-0.01606 & 0.8292 & 0.362492\end{array}$

$\begin{array}{llll}-0.01579 & 0.8030 & 0.370189\end{array}$

$\begin{array}{llll}-0.01528 & 0.7522 & 0.385789\end{array}$

$\begin{array}{llll}-0.01582 & 0.8061 & 0.369272\end{array}$

$\begin{array}{llll}-0.01900 & 1.1678 & 0.279862\end{array}$

$\begin{array}{llll}-0.02224 & 1.5648 & 0.210963\end{array}$

$\begin{array}{llll}-0.02468 & 1.7174 & 0.190033\end{array}$

$\begin{array}{llll}0.00951 & 0.4116 & 0.521137\end{array}$

$\begin{array}{llll}-0.01187 & 0.7027 & 0.401881\end{array}$

$\begin{array}{llll}0.00529 & 0.1330 & 0.715349\end{array}$

$\begin{array}{llll}-0.00672 & 0.2174 & 0.641015\end{array}$

$\begin{array}{lll}0.00942 & 0.4181 & 0.517906\end{array}$

$\begin{array}{llll}0.02533 & 3.3644 & 0.066619\end{array}$

$\begin{array}{llll}0.00402 & 0.0758 & 0.783052\end{array}$

NA $68.6535 \quad 0.000839$

\#\# Analysis of Deviance Table

\#\# Cox model: response is Surv(tstart, Tstop, status)

\#\# Model 1: pspline(iss.2, df = 7) + pspline(sbp.2, df = 5) + Sex.2:strata(t10group) + age10 + gcs.2

\#\# Model 2: pspline(iss.2, df = 5) + pspline(sbp.2, df = 5) + Sex.2:strata(t10group) + age10 + gcs.2

\#\# loglik Chisq Df $P(>\mid$ Chil)

\#\# $1-35986$

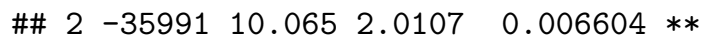

\#\# ---

\#\# Signif. codes: 0 '***' 0.001 '**' $0.011^{\prime} *$ ' 0.05 '.' 0.1 ' ' 1 
Final model for time to discharge outcome (Table 3)

\#Extract $H R$ with $95 \%$ CI

D3. $\mathrm{HR}=$ round $(\operatorname{summary}(\mathrm{D} 3) \$ \operatorname{conf}$. int $[, \mathrm{c}(1,3,4)], \operatorname{digits}=2)$

D3. HR

\#\#

\#\# ps (iss. 2) 3

\#\# ps (iss. 2) 4

\#\# ps (iss. 2) 5

\#\# ps (iss. 2) 6

\#\# ps (iss. 2) 7

\#\# ps(iss.2) 8

\#\# ps(iss.2) 9

\#\# ps (iss.2) 10

\#\# ps (iss.2) 11

\#\# ps(iss.2) 12

\#\# ps (iss.2) 13

\#\# ps (iss.2) 14

\#\# ps (iss.2) 15

\#\# ps (iss.2) 16

\#\# ps (iss.2) 17

\#\# ps (iss.2) 18

\#\# ps (iss.2) 19

\#\# ps (iss.2) 20

\#\# ps (iss. 2) 21

\#\# ps (iss. 2) 22

\#\# ps (sbp. 2) 3

\#\# ps (sbp. 2) 4

\#\# ps (sbp. 2) 5

\#\# ps (sbp.2) 6

\#\# ps (sbp.2) 7

\#\# ps (sbp.2)8

\#\# ps (sbp.2) 9

\#\# ps (sbp.2) 10

\#\# ps (sbp. 2) 11

\#\# ps (sbp. 2) 12

\#\# ps (sbp.2) 13

\#\# ps (sbp. 2) 14

\#\# ps (sbp. 2) 15

\#\# ps (sbp. 2) 16

\#\# age10

\#\# gcs. 2

\#\# ICED. 2

\#\# Sex.2:strata(t10group)t10

\#\# Sex.2:strata(t10group)t10

\#\# ICU.2:strata(timegroup)ti

\#\# ICU.2:strata(timegroup)ti

\#\# ICU.2:strata(timegroup)ti

$\begin{array}{crr}\exp \text { (coef) } & \text { lower } .95 & \text { upper } .95 \\ 1.13 & 0.71 & 1.81 \\ 1.10 & 0.66 & 1.84 \\ 0.77 & 0.47 & 1.28 \\ 0.66 & 0.40 & 1.09 \\ 0.74 & 0.45 & 1.24 \\ 0.79 & 0.48 & 1.32 \\ 0.67 & 0.40 & 1.13 \\ 0.59 & 0.35 & 1.01 \\ 0.55 & 0.32 & 0.94 \\ 0.51 & 0.29 & 0.89 \\ 0.43 & 0.24 & 0.78 \\ 0.40 & 0.21 & 0.75 \\ 0.41 & 0.20 & 0.85 \\ 0.40 & 0.17 & 0.98 \\ 0.36 & 0.12 & 1.04 \\ 0.29 & 0.09 & 1.00 \\ 0.23 & 0.06 & 0.88 \\ 0.18 & 0.04 & 0.80 \\ 0.14 & 0.02 & 0.89 \\ 0.11 & 0.01 & 1.25 \\ 1.07 & 0.57 & 2.01 \\ 1.14 & 0.39 & 3.38 \\ 1.24 & 0.31 & 4.96 \\ 1.38 & 0.29 & 6.51 \\ 1.52 & 0.30 & 7.58 \\ 1.65 & 0.33 & 8.19 \\ 1.86 & 0.38 & 9.20 \\ 1.91 & 0.39 & 9.44 \\ 1.74 & 0.35 & 8.61 \\ 1.64 & 0.33 & 8.13 \\ 1.53 & 0.31 & 7.59 \\ 1.44 & 0.29 & 7.24 \\ 1.35 & 0.25 & 7.30 \\ 1.26 & 0.19 & 8.34 \\ 0.98 & 0.96 & 0.99 \\ 1.04 & 1.02 & 1.06 \\ 0.79 & 0.73 & 0.86 \\ 0.98 & 0.91 & 1.05 \\ 0.79 & 0.71 & 0.87 \\ 0.39 & 0.33 & 0.46 \\ 0.60 & 0.52 & 0.71 \\ 0.88 & 0.66 & 1.18 \\ & & \\ 0.96 & \end{array}$

\#Extract $p$-value

D3. $P=$ round (summary (D3) \$coefficient $[, 6]$, digits = 3)

D3.P 


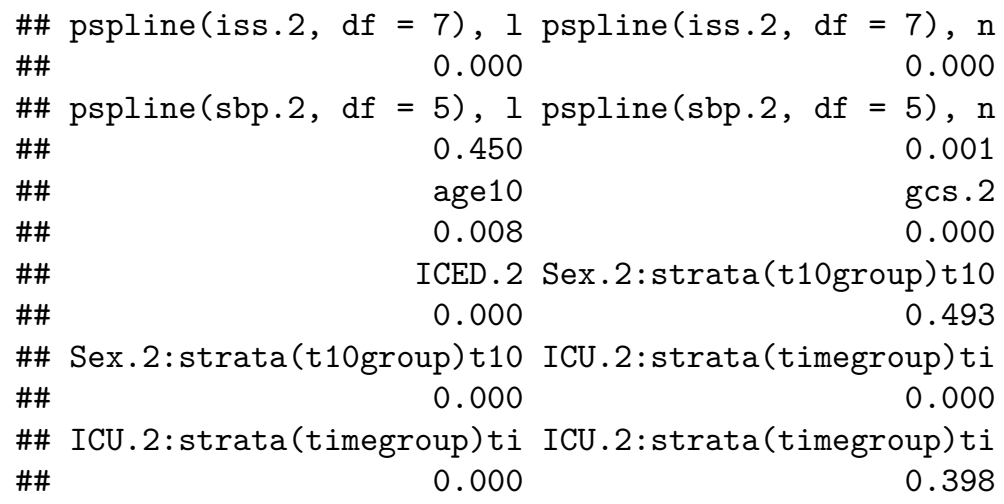

Nonlinear plot (Figure 2)

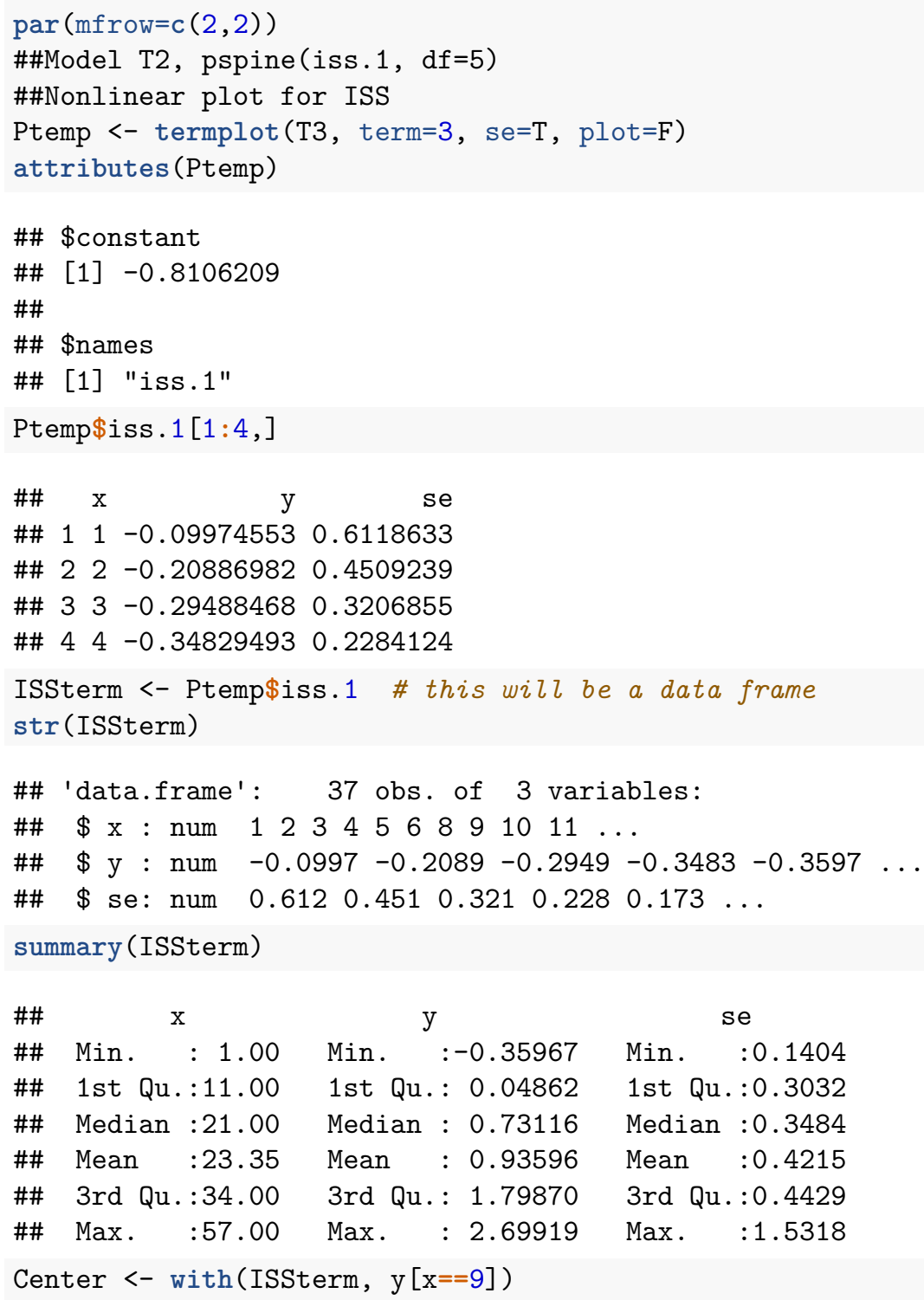




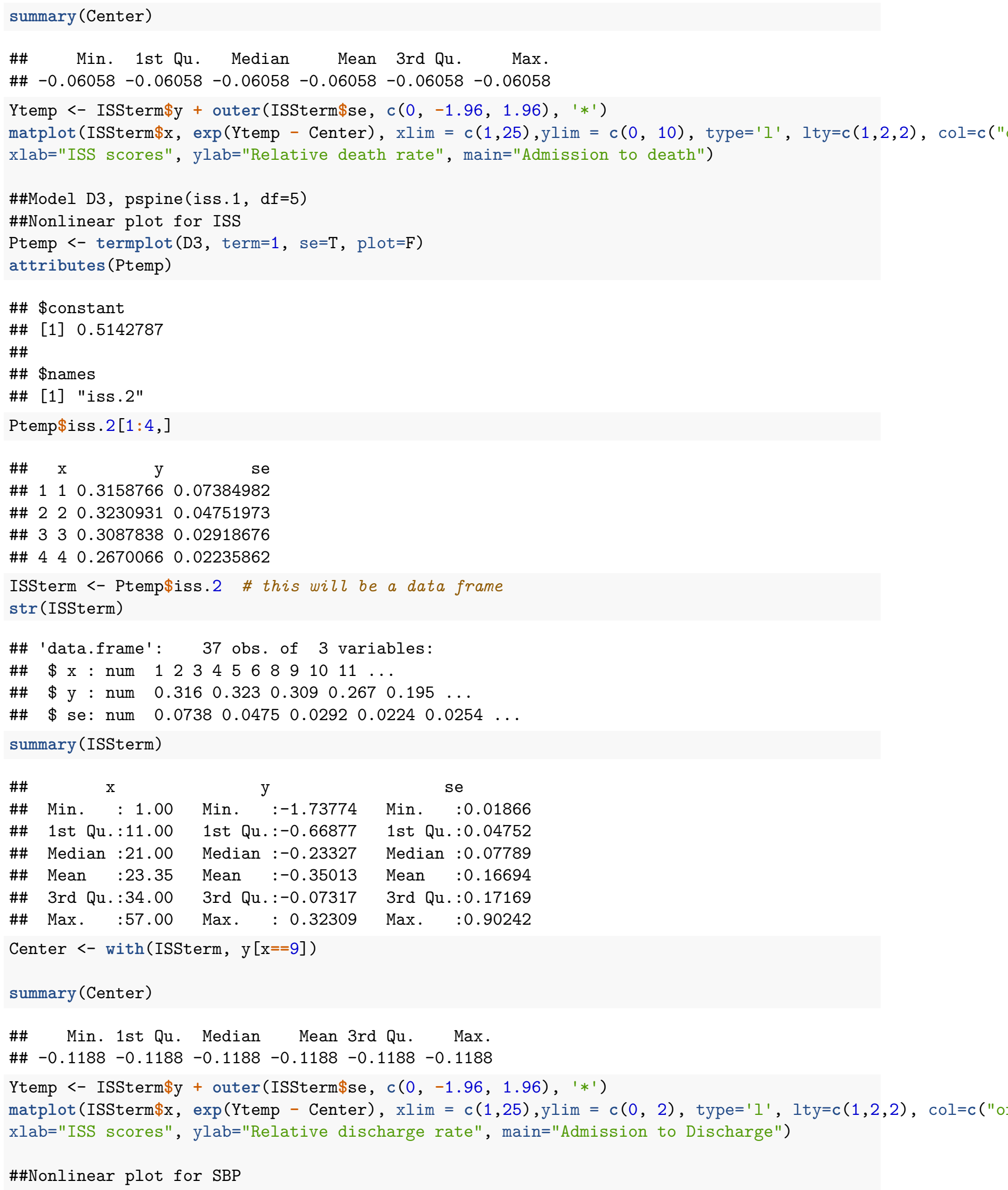




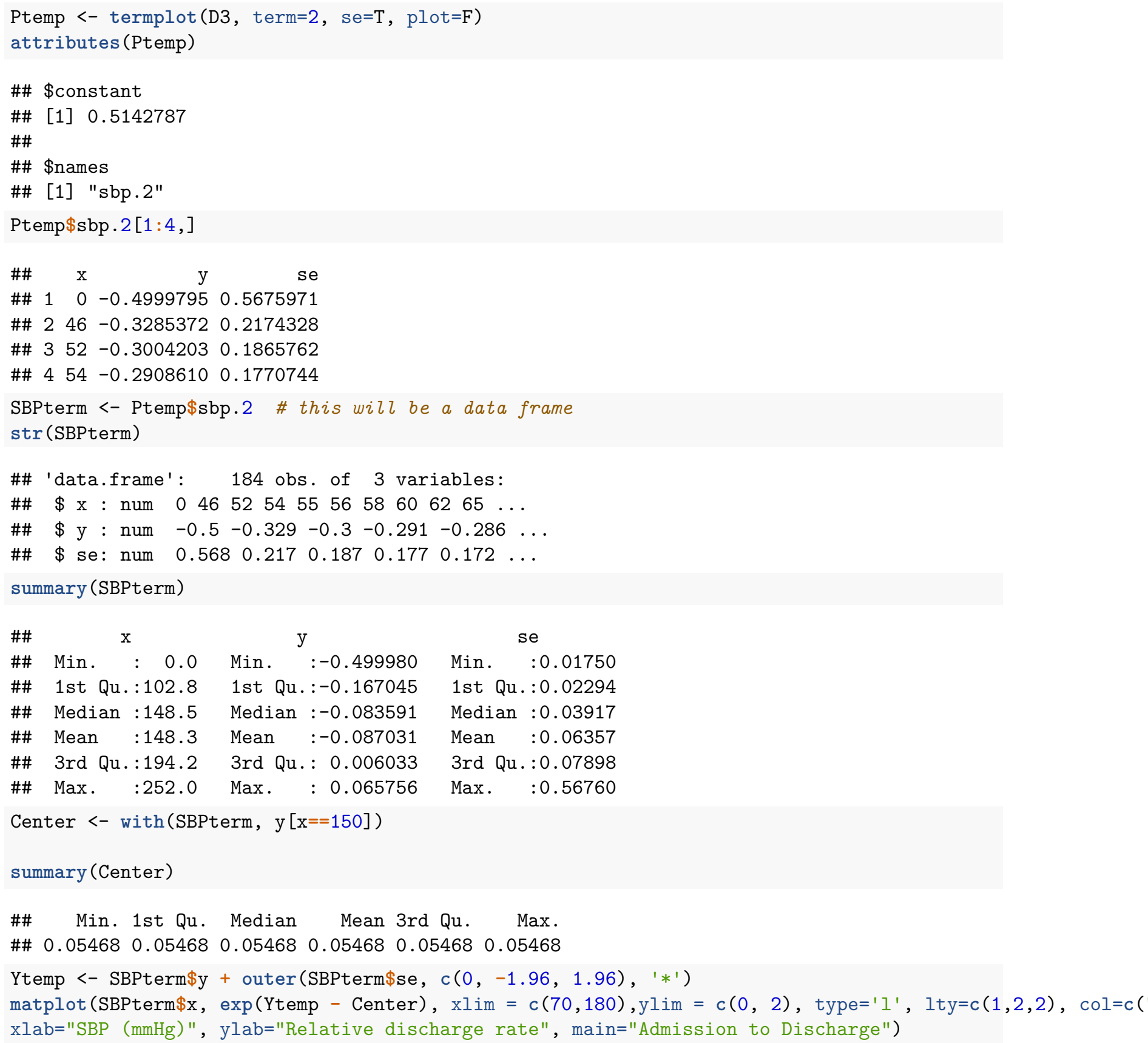



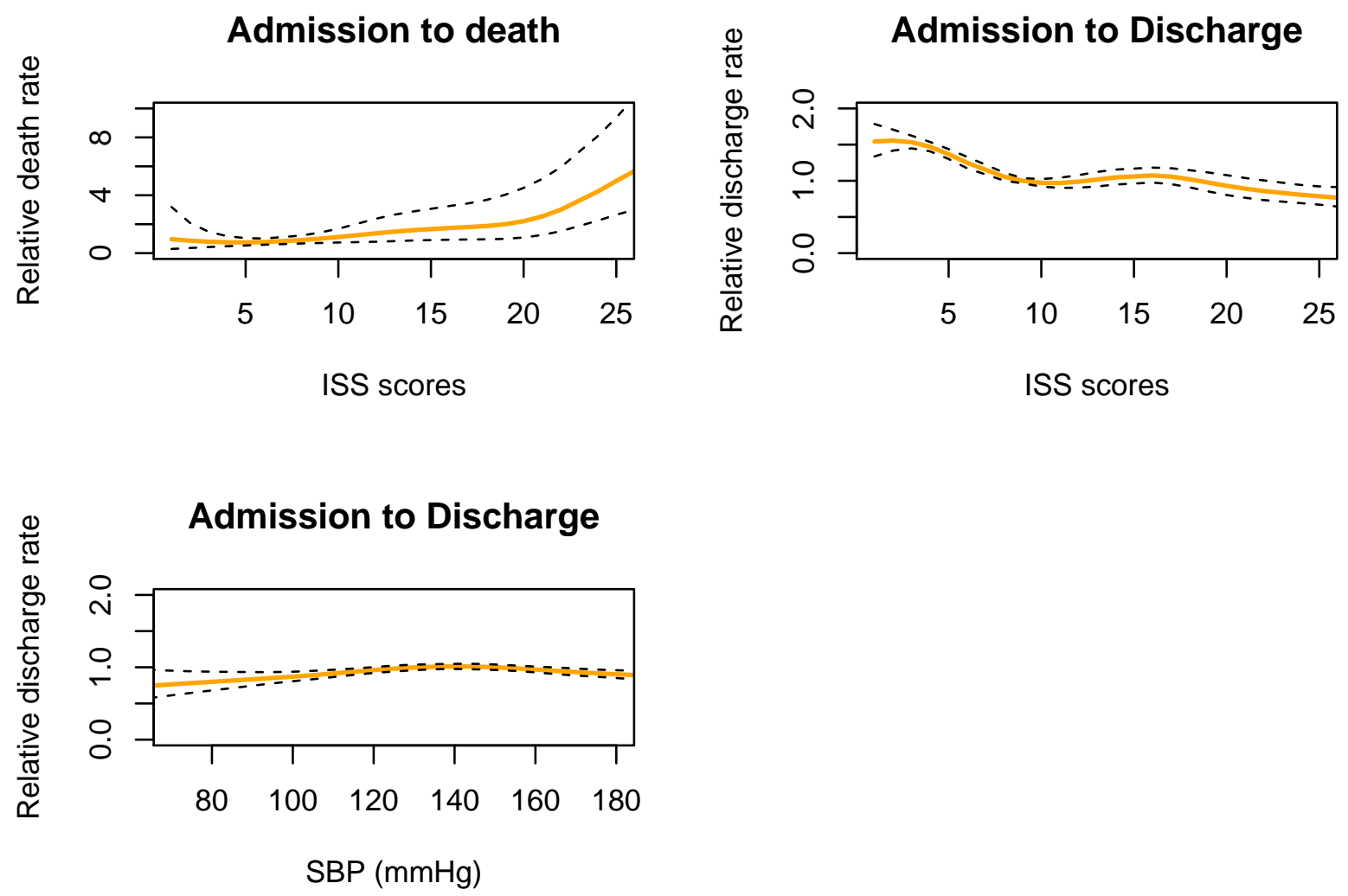

ISS scores stratified by sex

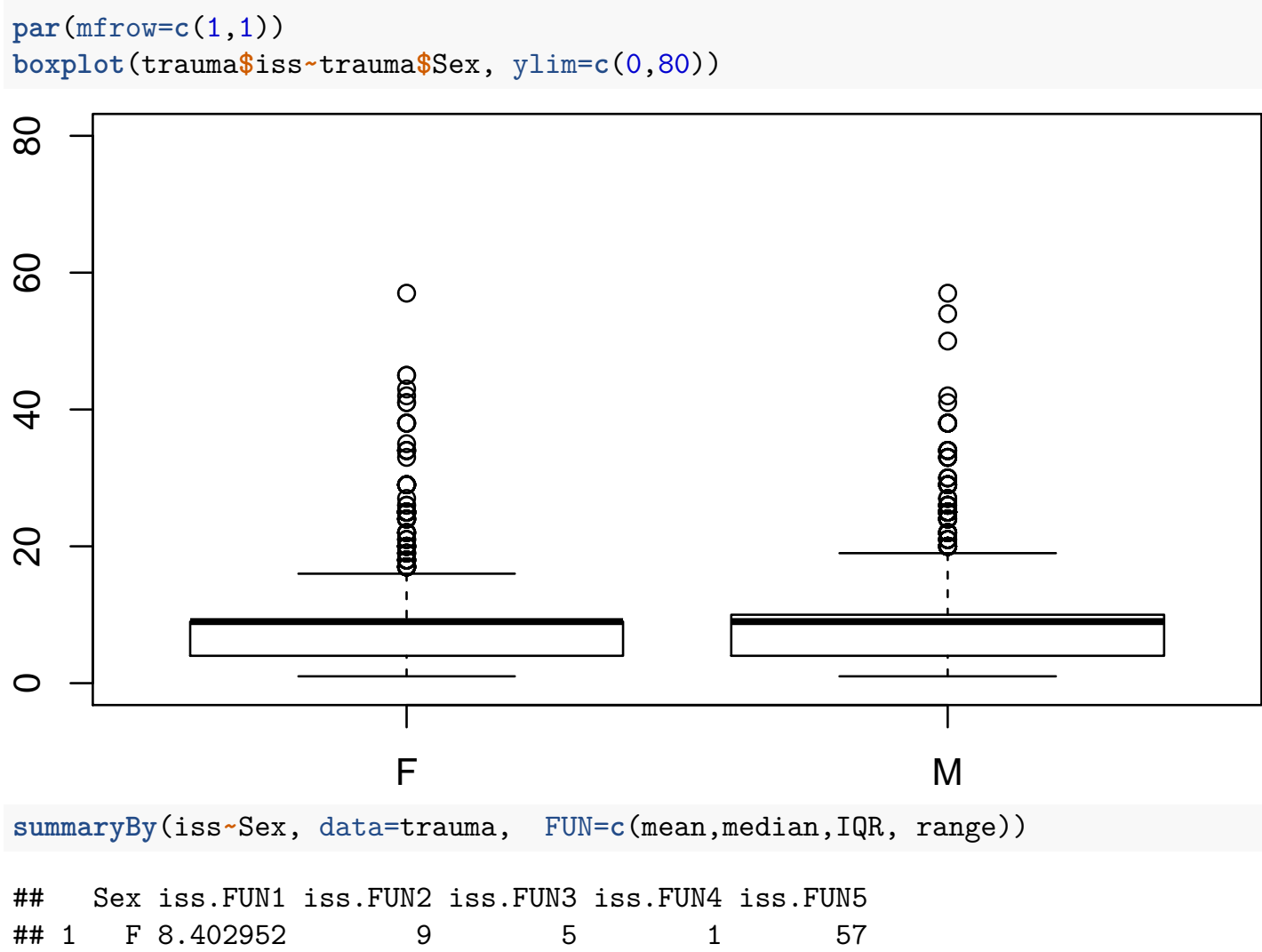




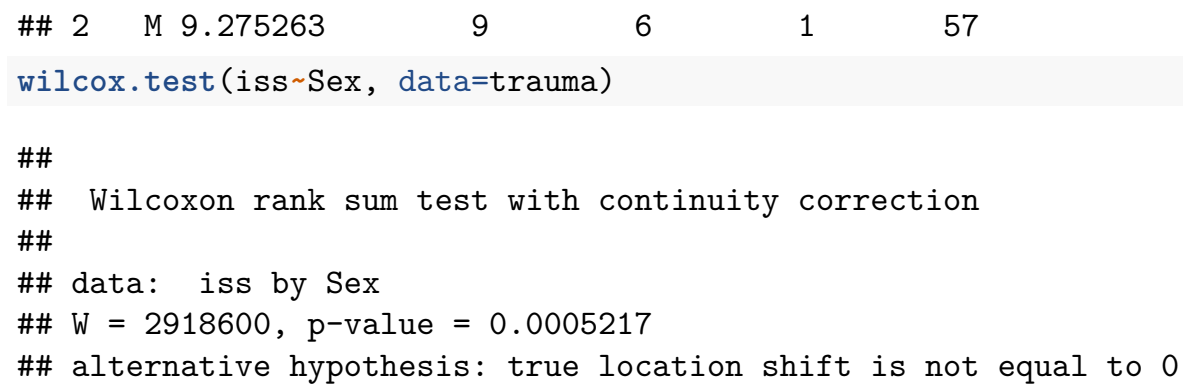

\section{Log-linear model for conditional independence}

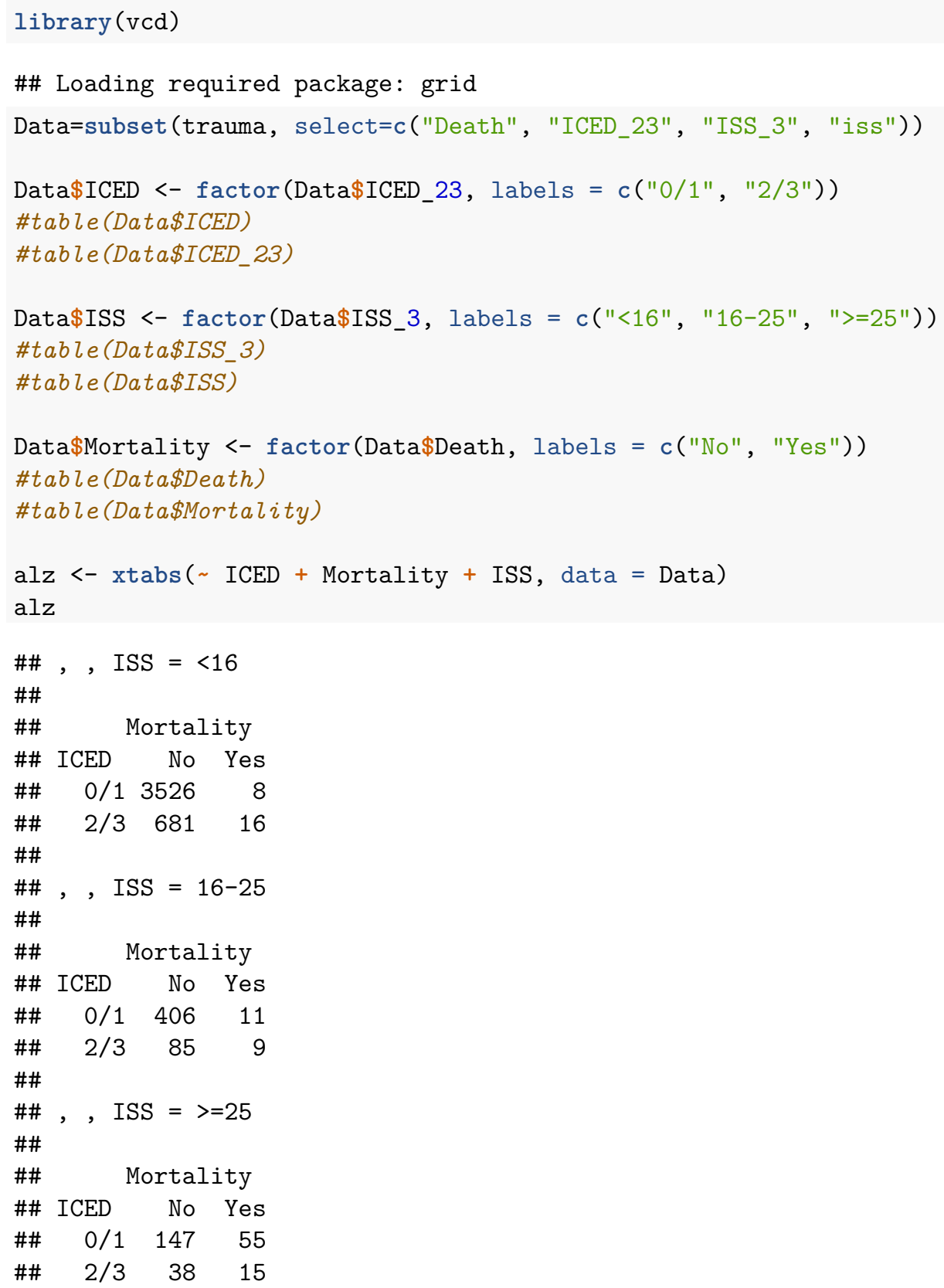


\#\#\#\#\#\#\#\#\#\#\#\#\#\#\#\#\#\#\#\#\#\#\#\#\#\#\#\#\#\#\#\#\#\#\#\#\#\# Mosaic plot conditional on ISS \#\#\#\#\#\#\#\#\#\#\#\#\#\#\#\#\#\#\#\#\#\#\#\#\#\#\#\#\#\#\#\#\#\#\#\#\#\#\#\#\#\#

rseed <- 1071

set.seed (rseed)

cotabplot ( ICED + Mortality | ISS, data $=$ alz, panel $=$ cotab_coindep, $\mathrm{n}=5000)$

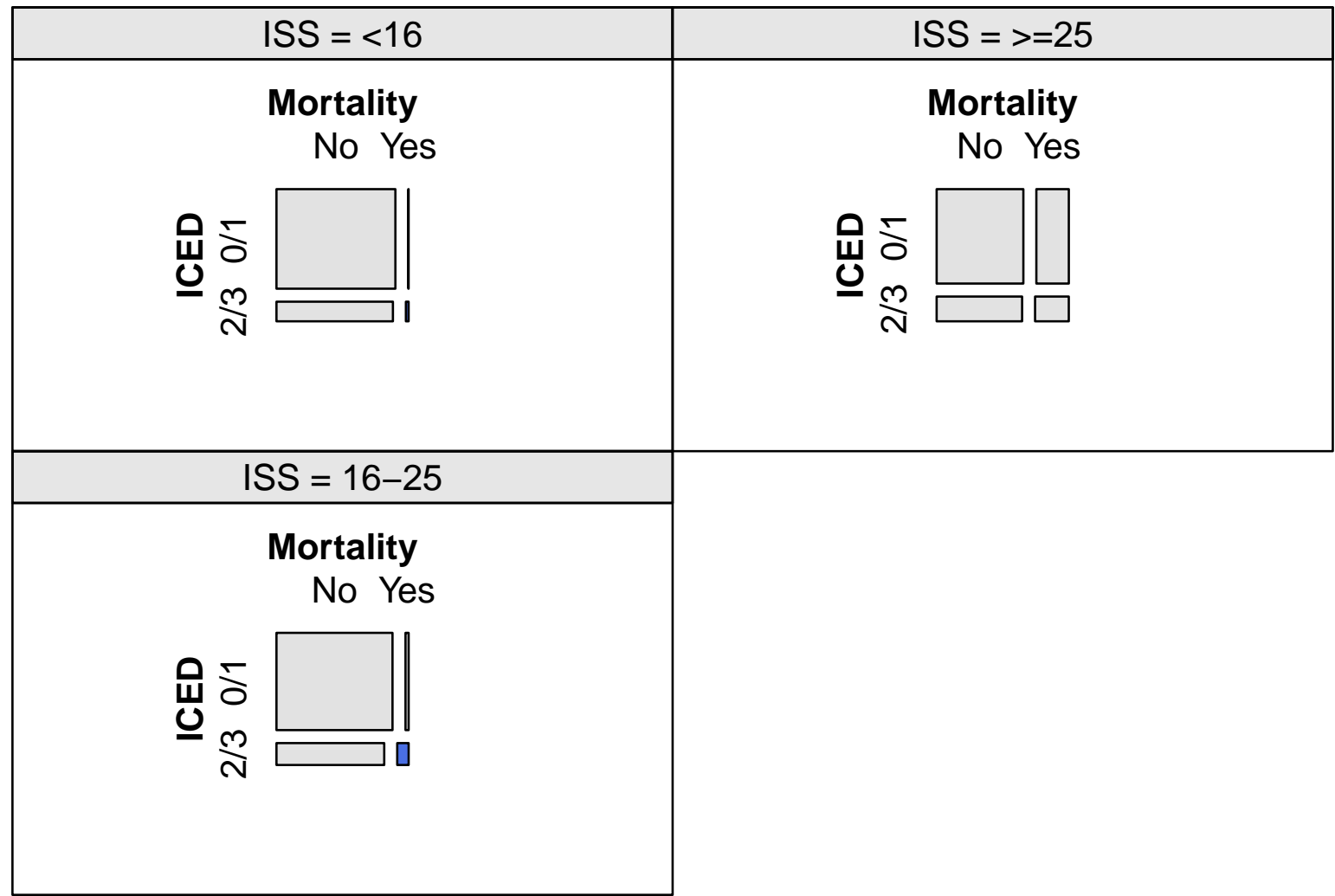

\#\#\#\#\#\#\#\#\#\#\#\#\#\#\#\#\#\#\#\#\#\#\#\#\#\#\#\#\#\#\#\#\#\#\#\#\#\#\#\#\#Check indepedence test for log-linear model using \#\#

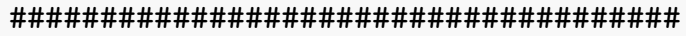

ss $<-\operatorname{function}(\mathrm{x}) \operatorname{sum}\left(\mathrm{x}^{\wedge} 2\right)$

set.seed (rseed)

coindep_test (alz, 3, $\mathrm{n}=5000$ )

\#\#

\#\# Permutation test for conditional independence

\#\#

\#\# data: alz

\#\# $f(x)=6.0583, p$-value $<2.2 e-16$

set.seed (rseed)

coindep_test $(\mathrm{alz}, 3, \mathrm{n}=5000$, indepfun $=\mathrm{ss})$

\#\#

\#\# Permutation test for conditional independence

\#\#

\#\# data: alz

\#\# $\mathrm{f}(\mathrm{x})=44.193, \mathrm{p}$-value $<2.2 \mathrm{e}-16$

set.seed (rseed)

coindep_test $(\mathrm{alz}, 3, \mathrm{n}=5000$, indepfun $=\mathrm{ss}, \operatorname{aggfun}=$ sum $)$ 
\#\# Permutation test for conditional independence

\#\#

\#\# data: alz

\#\# $f(x)=54.032, p$-value $<2.2 e-16$

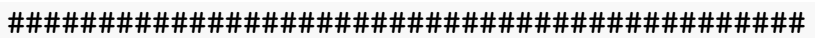

Split <- c(T,F, T)

\#\#HCL shading, Figure 3

mosaic(alz, expected $=\sim$ (Mortality + ICED) $*$ ISS,gp = shading_hcl, split_vertical = Split)

ICED

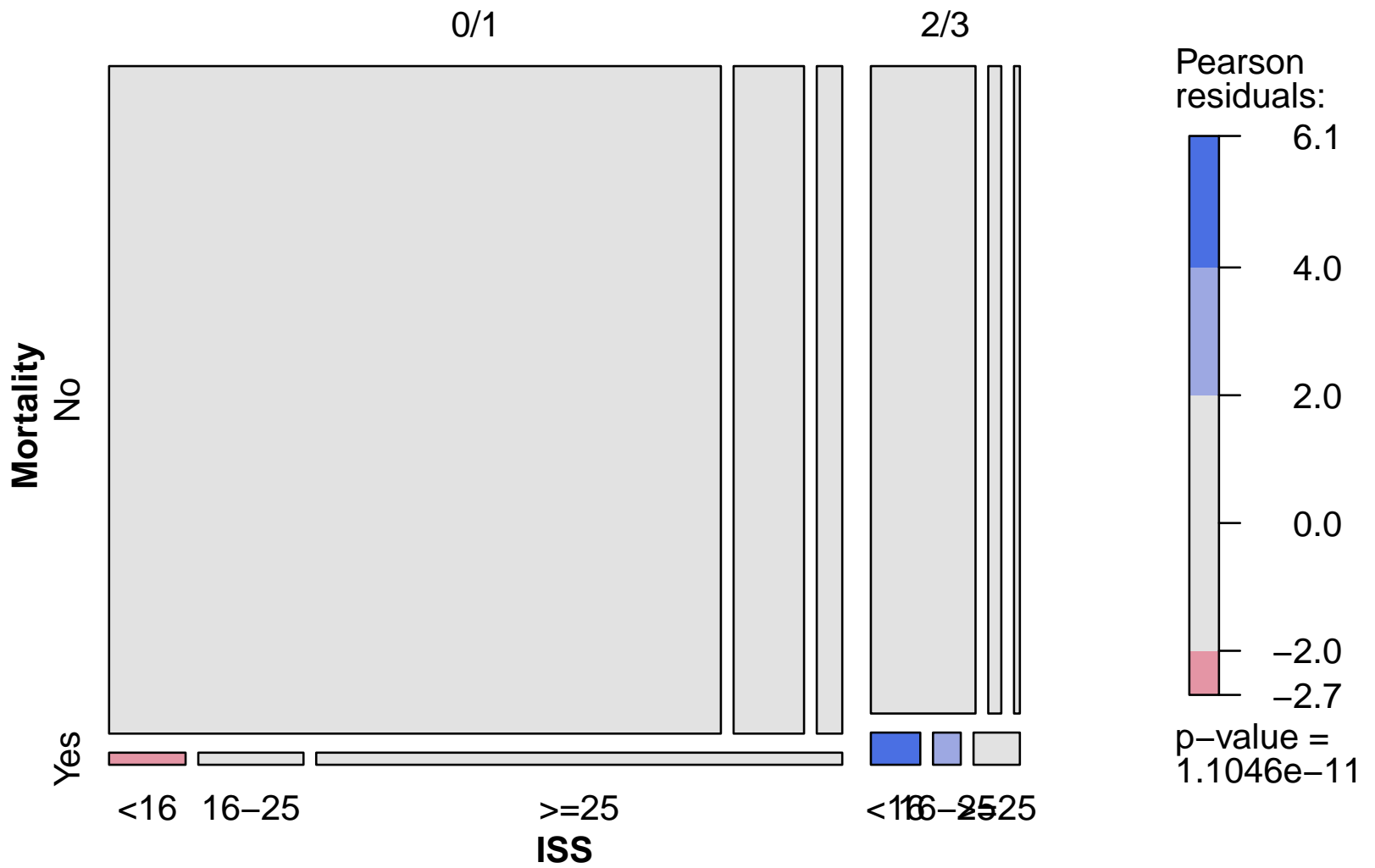

\#\#Fiendly shading

mosaic (alz, expected $=\sim$ (Mortality + ICED) $*$ ISS,gp = shading_Friendly, split_vertical = Split) 


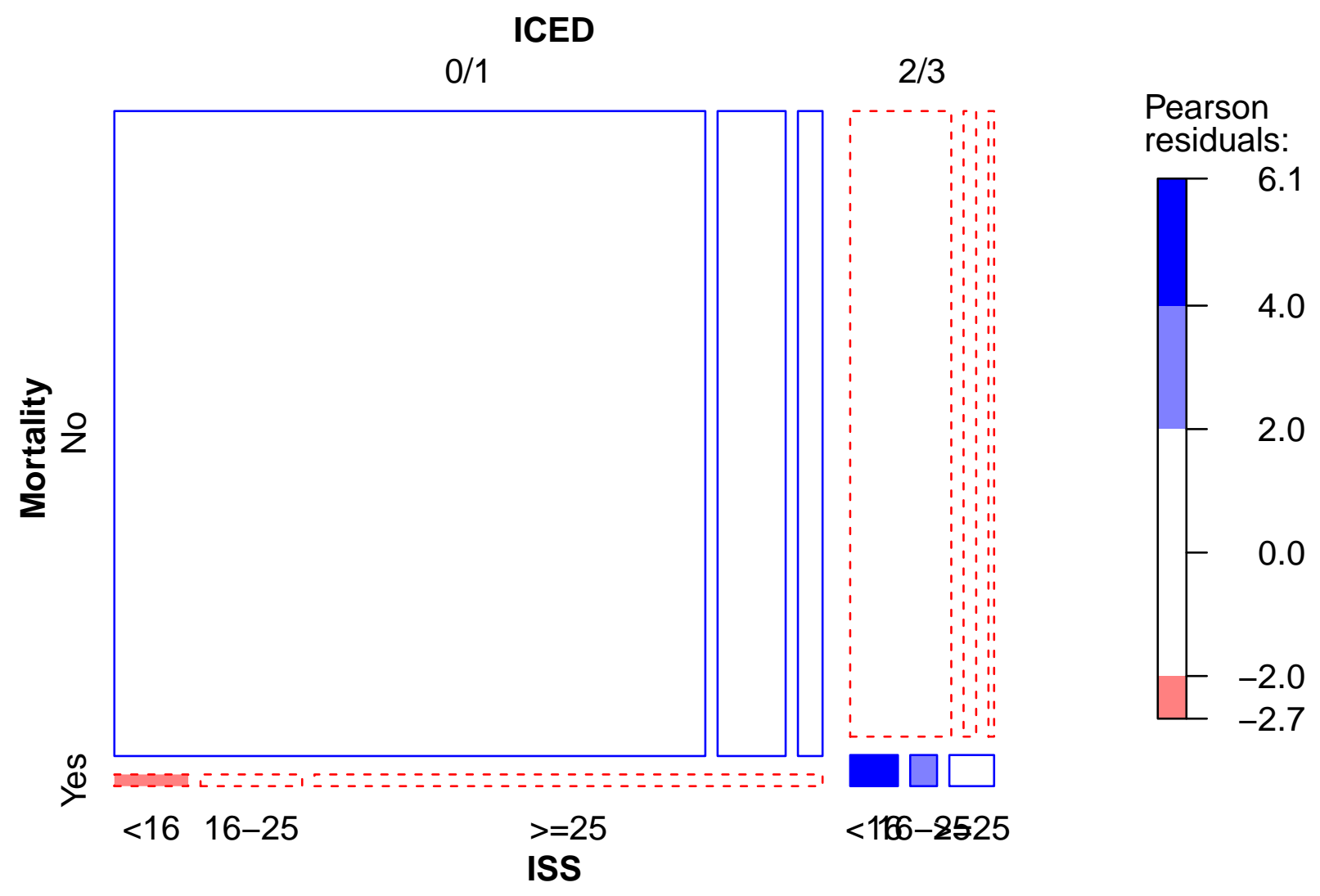

